
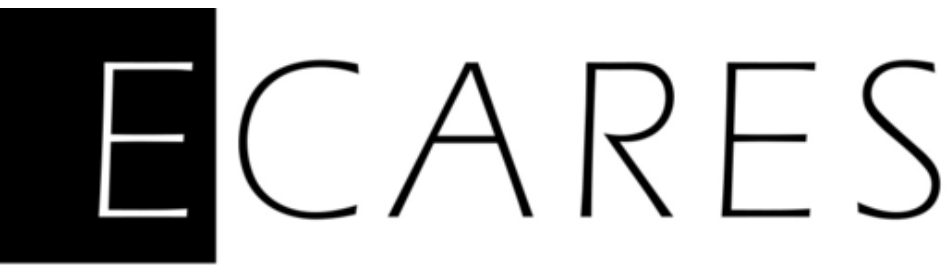

\title{
An Indirect Proof for the Asymptotic Properties of VARMA Model Estimators
}

ECARES, Université libre de Bruxelles

April 2020

ECARES working paper 2020-10 


\title{
An indirect proof for the asymptotic properties of VARMA model estimators ${ }^{\hat{2}}$
}

\author{
Guy Mélard ${ }^{\mathrm{a}, 1, *}$ \\ ${ }^{a}$ Av. Franklin Roosevelt 50 CP114/04, B-1050 Brussels, Belgium
}

\begin{abstract}
Strong consistency and asymptotic normality of a Gaussian quasi-maximum likelihood estimator for the parameters of a causal, invertible, and identifiable vector autoregressive-moving average (VARMA) model are established in an indirect way. The proof is based on similar results for a much wider class of VARMA models with time-dependent coefficients, hence in the context of non-stationary and heteroscedastic time series. For that reason, the proof avoids spectral analysis arguments and does not make use of ergodicity. The results presented are also applicable to ARMA models.

Declarations of interest: none Keywords: non-stationary process; multivariate time series; time-varying models; identifiability; ARMA models.
\end{abstract}

\footnotetext{
Supplementary Material entitled "From tdARMA models to ARMA models" is available for ARMA models.

* Corresponding author

Email address: gmelard@ulb.ac.be ( Guy Mélard )

${ }^{1}$ Université libre de Bruxelles, Faculty SBS-EM \& ECARES.
} 


\section{Introduction}

Vector autoregressive-moving average (VARMA) models are the multivariate generalization of univariate autoregressive-moving average (ARMA) models. ARMA models are used to represent stationary time series in almost all domains where a variable is measured at equidistant times. Its generalizations, ARIMA and SARIMA models, respectively for integrated and seasonal time series, can cope with a stochastic trend and stochastic seasonality. Vector autoregressive (VAR) models are widely used in econometrics. VARMA models are much less used, except in certain specific applications. For example, they are able to represent competitive behaviour between products in economics, see Jenkins (1979). VARMA models have been widely studied since the 1970's, starting with Hannan (1969) and Tiao and Box (1981). See the books by Hannan and Deistler (2012), first published in 1988, Reinsel (1997), Lütkepohl (2005), Wei (2006), and Tsay (2014). New theoretical results are still being developed for model specification (Poskitt, 2005; Athanasopoulos and Vahid, 2008a; Boubacar Maïnassara, 2012; Brockwell et al., 2012; Wegge, 2012), in particular in structural models (Gouriéroux et al. 2017, 2020), for estimation methods (Kascha, 2012; Boubacar Maïnassara and Francq, 2011; Dufour and Jouini, 2014; Hallin et al., 2020; Roy et al., 2019), also in the case of non-independent but uncorrelated errors (Boubacar Maïnassara and Saussereau, 2018; Dufour and Pelletier, 2019), for diagnostic checking (Boubacar Maïnassara, 2011), and for forecasting (Lütkepohl, 2006; Athanasopoulos and Vahid, 2008b; Kascha and Mertens, 2009). Work is also being carried out on sparse models (Wilms et al., 2019).

More recently, ARMA and VARMA models with time-dependent coefficients have been proposed and asymptotic theories have been developed in order to justify their use. In most cases, a parametric approach has been taken, but there are also semiparametric approaches. In parametric approaches, it is assumed that there is a relatively small number of parameters. In Alj et al. (2017), the authors have considered time-dependent VARMA (tdVARMA) models with possibly marginal heteroscedasticity (as opposed to conditional heteroscedasticity). Subject to a set of assumptions, they establish strong consistency and asymptotic normality of a Gaussian quasimaximum likelihood estimator (QMLE) for the parameters of a tdVARMA model.

We present a new proof of the asymptotic properties of standard, i.e. homoscedastic, causal, invertible, and identifiable VARMA models. That proof is indirect and is based on Alj et al. (2017) and a new representation of tdVARMA models under a higher-dimensional tdVAR(1) model. This is done, on the basis of Lütkepohl (1991), 
by combining features from the two papers of Francq and Gautier (2004) and Francq and Zakoïan (2001); see also Boubacar Maïnassara and Rabehasaina (2020). This makes it possible to check the assumptions made by Alj et al. (2017). It is, therefore, an indirect proof. The paper is of course also applicable to ARMA models (see the Supplementary Material).

Since the proof of the asymptotic properties for tdVARMA models does not exploit ergodicity, a property that is certainly invalid for tdVARMA models, which are not usually stationary, our indirect proof for VARMA model does not use ergodicity. Although there exist elements of spectral theory for non-stationary time series, the Alj et al. (2017) approach does not make use of spectral theory. Consequently, the indirect new proof of the asymptotic properties for VARMA models is provided entirely in the time domain and does not refer to a frequency domain approach. Existing proofs of asymptotic properties in time series models, e.g. Hannan (1973), reported and expanded in Brockwell and Davis (1991) and Yao and Brockwell (2006), for scalar processes, or Dunsmuir and Hannan (1976), Deistler et al. (1978), Rissanen and Caines (1979), Kohn (1979), Hannan et al. (1980), and Ling and McAleer (2003) (the latter with GARCH errors), for vector processes, all make use of ergodicity. Several of these proofs also make use of spectral arguments, starting with Hannan (1973), but not those of Yao and Brockwell (2006) or Rissanen and Caines (1979). Several other comparative aspects will be discussed later, including the ability to handle structural models, the assumptions made about errors and their moments, and the compactness of the parameter space. It should be clear that our results do not require weaker assumptions than previous results. For example, we need the existence of fourthorder moments for the errors, while only the existence of the variance is needed in Yao and Brockwell (2006) for scalar models. The requirement is fourth-order moments for Ling and McAleer (2003) in the wider context of VARMA-ARCH models.

The proof for VARMA models is relatively short; it should be noted, however, that the Alj et al. (2017) paper relies on a law of large numbers and a central limit theorem for martingales and a law of large number for mixingales, and requires a long technical appendix to establish the required lemmas. The latter paper's technical appendix is, however, not needed for scalar models, as the simpler theory for tdARMA models in Azrak and Mélard (2006) is then applicable. Moreover, in order to reduce the moment assumption on the model errors, the two papers need to be complemented by a result of Azrak and Mélard (2020) .

In Section 2, we introduce VARMA and tdVARMA models, and quasi-maximum 
likelihood estimators (QMLE), and we state the assumptions for homoscedastic tdVARMA models. Section 3 is devoted to preliminaries, where tdVARMA models are put into a $\operatorname{tdVAR}(1)$ form that allows for easier treatment of the derivatives of the pure tdVMA representation. Section 4 contains the assumptions we need for standard VARMA models, a few remarks on those assumptions, a theorem of consistency and asymptotic normality of the QMLE estimators of a VARMA model, and its proof, which consists in the verification of the assumptions of Alj et al. (2017). As promised, the proof does not exploit ergodicity (even if the process is ergodic, given the assumptions being made) and does not require spectral arguments.

\section{QMLE for a tdVARMA or a VARMA time series model}

We introduce an estimator of the parameters of a zero-mean $r$-dimensional vector mixed autoregressive-moving average (VARMA) model with time-dependent coefficients, denoted by tdVARMA. Then we particularize it to the standard VARMA model.

\subsection{Definitions and notations}

We denote the transpose of a vector or matrix by ${ }^{T}$. We denote by $\left\{x_{t} ; t=\right.$ $1,2, \ldots\}$ a stochastic process with values in $\mathbf{R}^{r}$, which is defined on a probability space $\left(\Omega, F, P_{\theta}\right)$, and whose distribution depends on a vector $\theta=\left(\theta_{1}, \ldots, \theta_{m}\right)^{T}$ of parameters, $\theta \in \Theta$, an open set of a Euclidean space $\mathbf{R}^{m}$. The true value of $\theta$ is supposed to be $\theta^{0}=\left(\theta_{1}^{0}, \ldots, \theta_{m}^{0}\right)^{T}$, an interior point of $\Theta$.

We denote by $E_{\theta}($.$) and E_{\theta}(. /$.$) , respectively, the expectation and the conditional$ expectation under $P_{\theta}$. Let $\left\{F_{t} ; t=1,2, \ldots\right\}$ be an increasing sequence of sub-sigma algebras of $F$ with $F_{t}$ generated by $\left\{x_{u} ; u=1,2, \ldots, t\right\}$ with $F_{0}=\{\emptyset, \Omega\}$ such that $x_{t}$ is measurable with respect to $F_{t}$, for each $t$.

The process $\left\{x_{t} ; t=1,2, \ldots\right\}$ is called a zero-mean $r$-dimensional vector mixed autoregressive-moving average process of order $(p, q)$ with time-dependent coefficients, and is denoted by tdVARMA $(p, q)$, if and only if it satisfies the equation

$$
x_{t}=\sum_{i=1}^{p} A_{i, t} x_{t-i}+g_{t} \epsilon_{t}+\sum_{j=1}^{q} B_{j, t} g_{t-j} \epsilon_{t-j},
$$

where $p \geq 0$ and $q \geq 0$ are integer constants, where $\left\{\epsilon_{t} ; t=1,2, \ldots\right\}$ is an independent white noise process, consisting of independent random vectors, not necessarily identically distributed, with a zero mean, a covariance matrix $\Sigma$ which is invertible, and finite moments of order 4 , and where the coefficients $A_{1, t}, \ldots, A_{p, t}$, and $B_{1, t}, \ldots, B_{q, t}$, as 
well as $g_{t}$, are $r \times r$ matrices with elements that are deterministic functions of time $t$. The initial values $x_{t}, t<1$, and $\epsilon_{t}, t<1$, are supposed to be equal to the zero vector. Writing $\otimes$ for the Kronecker product and $\operatorname{vec}($.$) for stacking the columns of a ma-$ trix on top of one another, we let $\kappa_{t}=E\left(\operatorname{vec}\left(\epsilon_{t} \epsilon_{t}^{T}\right) \operatorname{vec}\left(\epsilon_{t} \epsilon_{t}^{T}\right)^{T}\right)=E\left(\left(\epsilon_{t} \epsilon_{t}^{T}\right) \otimes\left(\epsilon_{t} \epsilon_{t}^{T}\right)\right)$, which depends on $t$, in general. For $k, l=0,1, \ldots$ and $k \neq l$, we consider the matrix $E\left(\operatorname{vec}\left(\epsilon_{t-k} \epsilon_{t-l}^{T}\right) \operatorname{vec}\left(\epsilon_{t-l} \epsilon_{t-k}^{T}\right)^{T}\right)=K_{r, r}(\Sigma \otimes \Sigma)$, which does not depend on $t, k$ or $l$, and where the $r^{2} \times r^{2}$ matrix $K_{r, r}$ is a commutation matrix (see Kollo and von Rosen, 2005, p. 79, or Lemma 4.2 of Appendix S2 of Alj et al., 2017).

Let us now consider the parametric model corresponding to (2.1), namely

$$
x_{t}=\sum_{i=1}^{p} A_{i, t}(\theta) x_{t-i}+e_{t}(\theta)+\sum_{j=1}^{q} B_{j, t}(\theta) e_{t-j}(\theta),
$$

where the $e_{t}(\theta)$ can be considered as the model residuals defined by

$$
e_{t}(\theta)=x_{t}-\widehat{x}_{t / t-1}(\theta) \quad \text { with } \quad \widehat{x}_{t / t-1}(\theta)=E_{\theta}\left(x_{t} / F_{t-1}\right)
$$

and $A_{i, t}(\theta)$, for $i=1, \ldots, p$, and $B_{j, t}(\theta)$, for $j=1, \ldots, q$, are the parametric coefficients. We suppose that they are three times differentiable with respect to $\theta$. Furthermore, the covariance matrix of $e_{t}(\theta)$ is parametrized as $\Sigma_{t}(\theta)={ }^{\operatorname{def}} E_{\theta}\left(e_{t}(\theta) e_{t}^{T}(\theta) / F_{t-1}\right)=$ $g_{t}(\theta) \Sigma g_{t}^{T}(\theta)$. For $\theta=\theta^{0}$, we have $A_{i, t}\left(\theta^{0}\right)=A_{i, t}, B_{j, t}\left(\theta^{0}\right)=B_{j, t}, g_{t}\left(\theta^{0}\right)=g_{t}, e_{t}\left(\theta^{0}\right)=$ $g_{t} \epsilon_{t}$ and $\Sigma_{t}={ }^{\operatorname{def}} \Sigma_{t}\left(\theta^{0}\right)=E\left(e_{t}\left(\theta^{0}\right) e_{t}^{T}\left(\theta^{0}\right)\right)=g_{t} \Sigma g_{t}^{T}$. We assume that $\theta$ contains all the parameters of interest to be estimated, those involved in the coefficients $A_{1, t}(\theta), \ldots$, $A_{p, t}(\theta), B_{1, t}(\theta), \ldots, B_{q, t}(\theta)$ and $g_{t}(\theta)$, but not the nuisance parameters in the scale factor matrix $\Sigma$, which are estimated separately.

In standard $\operatorname{VARMA}(p, q)$ models, the coefficients $A_{1}(\theta), \ldots, A_{p}(\theta), B_{1}(\theta), \ldots, B_{q}(\theta)$ do not depend on $t$, the parameters can be the elements of the coefficient matrices, and $g_{t}(\theta)=I_{r}$, the $r \times r$ identity matrix. The model is written

$$
x_{t}=\sum_{i=1}^{p} A_{i}(\theta) x_{t-i}+e_{t}(\theta)+\sum_{j=1}^{q} B_{j}(\theta) e_{t-j}(\theta)
$$

but time $t$ is allowed to vary from $-\infty$ to $+\infty$. For $\theta=\theta^{0}$, we have $A_{i}\left(\theta^{0}\right)=A_{i}, i=$ $1, \ldots, p, B_{j}\left(\theta^{0}\right)=B_{j}, j=1, \ldots, q, e_{t}\left(\theta^{0}\right)=\epsilon_{t}$. We also assume (see $\mathbf{B}_{1}$ in Section 4.1) identically distributed (i.i.d.) errors $\epsilon_{t}$, so $\kappa_{t}=\kappa$ for all $t$. The other assumptions are that $\left(\mathbf{B}_{2}\right)$ the matrix polynomials $A(z)=I_{r}-\sum_{i=1}^{p} A_{i} z^{i}$, and $\left(\mathbf{B}_{3}\right) B(z)=$ $I_{r}+\sum_{j=1}^{q} B_{j} z^{j}$ have all their eigenvalues (the roots of the determinantal equations $\operatorname{det}(A(z))=0$ and $\operatorname{det}(B(z))=0)$ outside of the unit circle of the complex disc, and 
$\left(\mathbf{B}_{4}\right)$ that two identifiability conditions are satisfied, i.e. $\left(\mathbf{B}_{4} \mathrm{i}\right)$ left coprimeness of $A(z)$ and $B(z)$, see below and in Section 4.7 , and $\left(\mathbf{B}_{4} \mathrm{ii}\right)$ an additional condition that the matrix obtained by the juxtaposition of $A_{p}$ and $B_{q}$ has full rank. The two conditions in $\left(\mathbf{B}_{2}-\mathbf{B}_{3}\right)$ are, respectively, the causality condition and the invertibility condition. The causality condition is not fulfilled if there are unit roots, so we will not consider that case. The invertibility condition allows the time series to be correctly predicted from the model. The conditions $\left(\mathbf{B}_{4}\right)$ are essential to obtain an asymptotic covariance matrix for the estimator, see Section 4.7. Left-coprimeness of $A(z)$ and $B(z)$ means that the greatest left common divisor of $A(z)$ and $B(z)$ is $I_{r}$. In the most general case where each element of each matrix is a parameter, we would have $p r^{2}+q r^{2}$ as the total number of parameters, since we treat the elements of $\Sigma$ as nuisance parameters.

Remark 2.1. VARMA models are generally considered for all $t \in \mathbb{Z}$ although the observations start at some finite time, 1 say. Here we consider a process starting at time 1. Under the conditions in $\left(\mathbf{B}_{2}-\mathbf{B}_{3}\right)$, the effect of the initial conditions (the zero vectors used for $x_{t}, t<1$, and $\epsilon_{t}, t<1$ ) is forgotten when $t \rightarrow \infty$. Remember that we are interested in asymptotic results, hence those when $n \rightarrow \infty$.

\subsection{Parameter estimation}

Let us come back to the general tdVARMA model. Given a sequence of observations $\left\{x_{t} ; t=1,2, \ldots, n\right\}$, we want to estimate $\theta^{0}$ by minimizing an objective function $Q_{n}(\theta)=Q_{n}\left(\theta ; x_{1}, \ldots, x_{n}\right)$ which depends on $\theta$ and the observations $\left\{x_{t} ; t=1,2, \ldots, n\right\}$. We use the Gaussian quasi-likelihood function $L_{n}\left(\theta ; x_{1}, \ldots, x_{n}\right)$, which is computed as if the process were Gaussian. It is given by

$$
L_{n}\left(\theta ; x_{1}, \ldots, x_{n}\right)=(2 \pi)^{-n r / 2} \prod_{t=1}^{n} \operatorname{det}\left(\Sigma_{t}(\theta)\right)^{-1 / 2} \exp \left\{-\frac{1}{2} \sum_{t=1}^{n} e_{t}^{T}(\theta) \Sigma_{t}^{-1}(\theta) e_{t}(\theta)\right\} .
$$

We take the objective function $Q_{n}(\theta)=-\log \left(L_{n}\left(\theta ; x_{1}, \ldots, x_{n}\right)\right)=(1 / 2) \sum_{t=1}^{n} \alpha_{t}(\theta)+$ $(r n / 2) \log (2 \pi)$, with $\alpha_{t}(\theta)=\log \left(\operatorname{det}\left(\Sigma_{t}(\theta)\right)\right)+e_{t}^{T}(\theta) \Sigma_{t}^{-1}(\theta) e_{t}(\theta)$. Then the QMLE of $\theta^{0}$ is defined as any measurable solution $\widehat{\theta}_{n}=\arg \min _{\theta \in \Theta} Q_{n}(\theta)$. Of course, the

objective function $Q_{n}(\theta)$ is twice continuously differentiable in $\theta$. Let $\widehat{\theta}_{n}$ be a sequence of estimators indexed by $n$.

\section{Preliminaries}

\subsection{Putting tdVARMA models in $t d V A R(1)$ form}

A representation of an ARMA model in $\operatorname{tdVAR}(1)$ form is not new (see Lütkepohl, 1991, and Lütkepohl, 2005). It was used by Francq and Gautier (2004) for tdARMA 
models and was detailed in a working paper by Francq and Gautier (2003). It was described there using a state-space representation. We will see that the state-space representation can be eliminated from the description and that VARMA models can be handled in the same way as ARMA models. Note that Francq and Zakoïan (2001) propose a similar technique for building a Markovian representation for Markov-switching VARMA models (see also Stelzer, 2009). See also Boubacar Maïnassara and Rabehasaina (2020). The purpose of these authors was to obtain a unique strictly stationary solution. In a sense, we combine features from those two papers by Francq and Gautier (2004) and Francq and Zakoïan (2001). Francq and Gautier (2004) also consider an intercept that we will ignore here. A similar $\operatorname{tdVAR}(1)$ form was used for the vector GARCH part of VARMA-GARCH models by Ling and McAleer (2003). See also Jeantheau (1998). We can start with the following equation, denoting the $(p+q) r \times 1$ vectors $X_{t}(\theta)=\left(x_{t}^{T}, x_{t-1}^{T}, \ldots, x_{t-p+1}^{T}, e_{t}^{T}(\theta), e_{t-1}^{T}(\theta), \ldots, e_{t-q+1}^{T}(\theta)\right)^{T}$ and $E_{t}(\theta)=\left(e_{t}^{T}(\theta), 0^{T}, \ldots, 0^{T}, e_{t}^{T}(\theta), 0^{T}, \ldots, 0^{T}\right)^{T}$, where the two occurrences of $e_{t}^{T}(\theta)$ are in positions 1 and $p+1$, and $0^{T}$ is an $1 \times r$ zero vector:

$$
X_{t}(\theta)=\phi_{t}(\theta) X_{t-1}(\theta)+E_{t}(\theta)
$$

where

$$
\phi_{t}(\theta)=\left(\begin{array}{cc}
A_{t}(\theta) & \tilde{B}_{t}(\theta) \\
O_{q, p} & C
\end{array}\right),
$$

and $A_{t}(\theta), \tilde{B}_{t}(\theta)$ and $C$ are respectively $p \times p, p \times q, q \times q$ block matrices with $r \times r$ blocks, and $O_{q, p}$ is a $q r \times p r$ zero matrix. It can be seen that $A_{t}(\theta), \tilde{B}_{t}(\theta)$ and $C$ have the following forms, with $0_{r}$ a $r \times r$ zero matrix,

$$
\begin{gathered}
A_{t}(\theta)=\left(\begin{array}{ccccc}
A_{1, t}(\theta) & A_{2, t}(\theta) & \ldots & A_{p-1, t}(\theta) & A_{p, t}(\theta) \\
I_{r} & 0_{r} & \ldots & 0_{r} & 0_{r} \\
0_{r} & I_{r} & \ldots & 0_{r} & 0_{r} \\
\vdots & \vdots & \ddots & \vdots & \vdots \\
0_{r} & 0_{r} & \ldots & I_{r} & 0_{r}
\end{array}\right), \\
\tilde{B}_{t}(\theta)=\left(\begin{array}{ccccc}
B_{1, t}(\theta) & B_{2, t}(\theta) & \ldots & B_{q-1, t}(\theta) & B_{q, t}(\theta) \\
0_{r} & 0_{r} & \ldots & 0_{r} & 0_{r} \\
0_{r} & 0_{r} & \ldots & 0_{r} & 0_{r} \\
\vdots & \vdots & \ddots & \vdots & \vdots \\
0_{r} & 0_{r} & \ldots & 0_{r} & 0_{r}
\end{array}\right),
\end{gathered}
$$




$$
C=\left(\begin{array}{ccccc}
0_{r} & 0_{r} & \ldots & 0_{r} & 0_{r} \\
I_{r} & 0_{r} & \ldots & 0_{r} & 0_{r} \\
0_{r} & I_{r} & \ldots & 0_{r} & 0_{r} \\
\vdots & \vdots & \ddots & \vdots & \vdots \\
0_{r} & 0_{r} & \ldots & I_{r} & 0_{r}
\end{array}\right)
$$

Note that $A_{t}(\theta)$ in $(3.3)$ is what is called a companion matrix (e.g. Pollock (1999), Reinsel (1997)) of the monic matrix polynomial $A_{t}^{*}(z, \theta)=z^{p}-\sum_{i=1}^{p} A_{i, t}(\theta) z^{p-i}$ which is the reciprocal polynomial of the autoregressive polynomial $A_{t}(z, \theta)=I_{r}-$ $\sum_{i=1}^{p} A_{i, t}(\theta) z^{i}$, i.e. $A_{t}^{*}(z, \theta)=z^{p} A_{t}\left(z^{-1}, \theta\right)$, because the $(p-1) \times p$ block matrix below the block row of the $p$ coefficients is composed by an $I_{(p-1) r}$ matrix bounded to the right by a block column of $0_{r}$. Let $A_{t}(z)=A_{t}\left(z, \theta^{0}\right)$. The matrix $\tilde{B}_{t}(\theta)$ defined in (3.4) is not a companion matrix.

For $\theta=\theta^{0}$, the true value of the parameter vector, we have $e_{t}\left(\theta^{0}\right)=\epsilon_{t}$, and we denote $\phi_{t}^{0}={ }^{\text {def }} \phi_{t}\left(\theta^{0}\right)$ in $(3.2)$, with its upper blocks $A_{t}^{0}=A_{t}\left(\theta^{0}\right)$ and $\tilde{B}_{t}^{0}=\tilde{B}_{t}\left(\theta^{0}\right)$, and

$$
X_{t}^{0}=X_{t}\left(\theta^{0}\right)=\phi_{t}^{0} X_{t-1}\left(\theta^{0}\right)+E_{t}^{0},
$$

where

$$
E_{t}^{0}=E_{t}\left(\theta^{0}\right)=\left(\epsilon_{t}^{T}, 0^{T}, \ldots, 0^{T}, \epsilon_{t}^{T}, 0^{T}, \ldots, 0^{T}\right)^{T}
$$

and the second $\epsilon_{t}^{T}$ is in position $p+1$. We can develop the recurrence (3.6) as a moving average

$$
X_{t}^{0}=\sum_{k=0}^{t-1} \Psi_{t k} E_{t-k}^{0} \quad \text { with } \Psi_{t k}=\left(\prod_{j=0}^{i-1} \phi_{t-j}^{0}\right),
$$

with the convention that $\prod_{\ell=0}^{-1}()=.I_{(p+q) r}$. The equations 3.1 and 3.8 were proposed by Lütkepohl 1991, Section 6.3.2). From there Francq and Gautier (2003) build a state-space representation and derive the following from it. We skip the state-space stage which is not relevant here. We introduce the coefficients $\psi_{t k}=U_{1} \Psi_{t k}\left(U_{1}^{T}+U_{p+1}^{T}\right)$, where $U_{p+1}$ is a $(p+q) r \times r$ matrix of the form $\left(\begin{array}{lllllll}0_{r} & \ldots & 0_{r} & I_{r} & 0_{r} & \ldots & 0_{r}\end{array}\right)^{T}$, with $I_{r}$ in block $(p+1)$, and $U_{1}$ is a $(p+q) r \times r$ matrix of the form $\left(\begin{array}{llll}I_{r} & 0_{r} & \ldots & 0_{r}\end{array}\right)^{T}$, with $I_{r}$ in block 1 . There are the coefficients in the pure MA representation of the process:

$$
x_{t}=\sum_{k=0}^{t-1} \psi_{t k} \epsilon_{t-k}
$$

Next, we introduce the block $(p+q) \times 1$ vector $\tilde{X}_{t}=\left(x_{t}^{T}, 0^{T}, \ldots, 0^{T}, x_{t}^{T}, 0^{T}, \ldots\right.$, $\left.0^{T}\right)^{T}$, where the only non-zero elements $x_{t}^{T}$ are in positions 1 and $p+1$. Let $K$ be a 
$(p+q) \times(p+q)$ block matrix with $0_{r}$ everywhere except the blocks in positions $(1,1)$ and $(p+1,1)$, which are equal to $I_{r}$. Then

$$
\tilde{X}_{t}=K X_{t}\left(\theta^{0}\right)=K X_{t}^{0}
$$

Let $J$ be a matrix based on a $(p+q) r \times(p+q) r$ identity matrix where the blocks $I_{r}$ at positions $(1,1)$ and $(p+1, p+1)$ are replaced by $0_{r}$ and the block $0_{r}$ in position $(p+1,1)$ is replaced by $-I_{r}$. Let us denote $F_{t}(\beta)=J \phi_{t}(\theta)$. It can be checked that

$$
F_{t}(\beta)=J \phi_{t}(\theta)=\left(\begin{array}{cc}
\tilde{C} & O_{p, q} \\
\tilde{A}_{t}(\theta) & B_{t}(\theta)
\end{array}\right)
$$

where the $q \times p$ block matrix $\tilde{A}_{t}(\theta)$ has the same first block row as $-A_{t}(\theta)$ but the other block rows contain $0_{r}$, the matrix $B_{t}(\theta)$ is the companion matrix of the monic matrix polynomial $B_{t}^{*}(z, \theta)=z^{q}+\sum_{j=1}^{q} B_{j, t}(\theta) z^{q-j}$ which is the reciprocal polynomial of the moving average polynomial $B_{t}(z, \theta)=I_{r}+\sum_{j=1}^{q} B_{j, t}(\theta) z^{j}$,

$$
B_{t}(\theta)=\left(\begin{array}{ccccc}
-B_{1, t}(\theta) & -B_{2, t}(\theta) & \ldots & -B_{q-1, t}(\theta) & -B_{q, t}(\theta) \\
I_{r} & 0_{r} & \ldots & 0_{r} & 0_{r} \\
0_{r} & I_{r} & \ldots & 0_{r} & 0_{r} \\
\vdots & \vdots & \ddots & \vdots & \vdots \\
0_{r} & 0_{r} & \ldots & I_{r} & 0_{r}
\end{array}\right)
$$

and $\tilde{C}$ is a block $p \times p$ matrix with a form similar to $C$ in 3.5$)$. Let $B_{t}(z)=B_{t}\left(z, \theta^{0}\right)$.

From (3.1) and (3.11), it is easy to check directly the identity

$$
X_{t}(\theta)=\tilde{X}_{t}+F_{t}(\theta) X_{t-1}(\theta)
$$

Solving the recurrence equation $(3.13)$ yields

$$
\begin{aligned}
X_{t}(\theta) & =\tilde{X}_{t}+F_{t}(\theta) \tilde{X}_{t-1}+F_{t}(\theta) F_{t-1}(\theta) \tilde{X}_{t-2}+F_{t}(\theta) F_{t-1}(\theta) F_{t-2}(\theta) \tilde{X}_{t-3}+\ldots \\
& =\sum_{k=0}^{t-1}\left(\prod_{\ell=0}^{k-1} J \phi_{t-\ell}(\theta)\right) \tilde{X}_{t-k}
\end{aligned}
$$

Given 3.10, we can now replace $\tilde{X}_{t-k}$ in 3.14 by $K X_{t-k}^{0}$ and then $X_{t-k}^{0}$ using (3.8)

$$
X_{t}(\theta)=\sum_{k=0}^{t-1}\left(\prod_{\ell=0}^{k-1} J \phi_{t-\ell}(\theta)\right) K\left\{\sum_{i=0}^{t-k-1}\left(\prod_{j=0}^{i-1} \phi_{t-k-j}^{0}\right) E_{t-k-i}^{0}\right\}
$$


Alternatively, we have the explicit moving average representation

$$
X_{t}(\theta)=\sum_{k=0}^{t-1}\left\{\sum_{s=0}^{k}\left(\prod_{\ell=0}^{k-s-1} J \phi_{t-\ell}(\theta)\right) K\left(\prod_{j=0}^{s-1} \phi_{t-k+s-j}^{0}\right)\right\} E_{t-k}^{0} .
$$

Hence

$$
X_{t}(\theta)=\sum_{k=0}^{t-1} \Psi_{t k}(\theta) E_{t-k}^{0}
$$

with

$$
\Psi_{t k}(\theta)=\sum_{s=0}^{k}\left\{\left(\prod_{\ell=0}^{k-s-1} J \phi_{t-\ell}(\theta)\right) K\left(\prod_{j=0}^{s-1} \phi_{t-k+s-j}^{0}\right)\right\} .
$$

Note that $\Psi_{t k}\left(\theta^{0}\right)=\Psi_{t k}$ defined in (3.8). Note also that the $(p+1)$ th block of $X_{t}(\theta)$ is $e_{t}(\theta)$. Given (3.7), 3.15) implies an expression for $e_{t}(\theta)$ in function of the errors $\epsilon_{t-k}, k=0, \ldots, t-1$ :

$$
e_{t}(\theta)=\sum_{k=0}^{t-1} \psi_{t 0 k}(\theta) \epsilon_{t-k}
$$

where the coefficients $\psi_{t 0 k}(\theta), k=0, \ldots, t-1$, are well defined as follows

$$
\psi_{t 0 k}(\theta)=U_{p+1}^{T} \Psi_{t k}(\theta) U_{1}
$$

\subsection{Derivatives with respect to parameters}

In the previous subsection, we obtained a moving average representation of the process in $\operatorname{tdVAR}(1)$ form. This is not enough for the asymptotic theory because we also need the coefficients $\psi_{t i k}(\theta), \psi_{t i j k}(\theta)$, and $\psi_{t i j \ell k}(\theta)$ of moving average representations analogous to 3.17

$$
\begin{aligned}
\frac{\partial e_{t}(\theta)}{\partial \theta_{i}} & =\sum_{k=1}^{t-1} \psi_{t i k}(\theta) \epsilon_{t-k} \\
\frac{\partial^{2} e_{t}(\theta)}{\partial \theta_{i} \partial \theta_{j}} & =\sum_{k=1}^{t-1} \psi_{t i j k}(\theta) \epsilon_{t-k}, \\
\frac{\partial^{3} e_{t}(\theta)}{\partial \theta_{i} \partial \theta_{j} \partial \theta_{\ell}} & =\sum_{k=1}^{t-1} \psi_{t i j \ell k}(\theta) \epsilon_{t-k}
\end{aligned}
$$

$i, j, \ell=1, \ldots, m$. Following Francq and Gautier (2003) again, these coefficients are obtained simply by taking derivatives of (3.17) with respect to one, two, or three 
elements of $\theta$ :

$$
\psi_{t i k}(\theta)=\frac{\partial \psi_{t 0 k}(\theta)}{\partial \theta_{i}}, \quad \psi_{t i j k}(\theta)=\frac{\partial^{2} \psi_{t 0 k}(\theta)}{\partial \theta_{i} \partial \theta_{j}}, \quad \psi_{t i j \ell k}(\theta)=\frac{\partial^{3} \psi_{t 0 k}(\theta)}{\partial \theta_{i} \partial \theta_{j} \partial \theta_{\ell}},
$$

$k=0, \ldots, t-1$. From $(3.18)$, we have, for example,

$$
\Psi_{t i k}(\theta)=\frac{\partial \Psi_{t k}(\theta)}{\partial \theta_{i}}, \quad \psi_{t i k}(\theta)=U_{p+1}^{T} \Psi_{t i k}(\theta) U_{1} .
$$

$i=1, \ldots, m$, and similar expressions for the other two derivatives in (3.20) and (3.21). We introduce $\psi_{t i k}=\psi_{t i k}\left(\theta^{0}\right), \psi_{t i j k}=\psi_{t i j k}\left(\theta^{0}\right), \psi_{t i j l k}=\psi_{t i j l k}\left(\theta^{0}\right), \Psi_{t i k}=\Psi_{t i k}\left(\theta^{0}\right)$, etc.

\subsection{The asymptotic properties in the homoscedastic case}

We adapt the assumptions of Alj et al. (2017) for homoscedastic tdVARMA models with i.i.d. errors, i.e. when $g_{t}(\theta)=I_{r}$, as this is what we need for standard VARMA models.

Using the Frobenius norm $\|A\|_{F}=\sqrt{\operatorname{tr}\left(A^{T} A\right)}$ for a $m \times n$ matrix $A$, we assume for all $t \in \mathbf{N}$ :

$\mathbf{A}_{1}$ : The matrices $A_{i, t}(\theta), i=1, \ldots, p$ and $B_{j, t}(\theta), j=1, \ldots, q$, are three times continuously differentiable with respect to $\theta$, in an open set $\Theta$ which contains the true value $\theta^{0}$ of $\theta$;

$\mathbf{A}_{2}$ : There exist positive constants $N_{\ell}, \ell=1, \ldots, 7$, and $0<\Phi<1$ such that, for $\nu=1, \ldots, t-1$, and $i, j, l=1, \ldots, m$,

$$
\begin{aligned}
& \sum_{k=\nu}^{t-1}\left\|\psi_{t i k}\right\|_{F}^{2}<N_{1} \Phi^{\nu-1}, \quad \sum_{k=\nu}^{t-1}\left\|\psi_{t i k}\right\|_{F}^{4}<N_{2} \Phi^{\nu-1}, \\
& \sum_{k=\nu}^{t-1}\left\|\psi_{t i j k}\right\|_{F}^{2}<N_{3} \Phi^{\nu-1}, \quad \sum_{k=\nu}^{t-1}\left\|\psi_{t i j k}\right\|_{F}^{4}<N_{4} \Phi^{\nu-1}, \\
& \sum_{k=1}^{t-1}\left\|\psi_{t i j l k}\right\|_{F}^{2}<N_{5}, \quad \sum_{k=1}^{t-1}\left\|\psi_{t k}\right\|_{F}^{2}<N_{6}, \quad \text { and } \quad \sum_{k=1}^{t-1}\left\|\psi_{t k}\right\|_{F}^{4}<N_{7} ;
\end{aligned}
$$

$\mathbf{A}_{3}: \kappa=E\left(\operatorname{vec}\left(\epsilon_{t} \epsilon_{t}^{T}\right) \operatorname{vec}\left(\epsilon_{t} \epsilon_{t}^{T}\right)^{T}\right)=E\left(\left(\epsilon_{t} \epsilon_{t}^{T}\right) \otimes\left(\epsilon_{t} \epsilon_{t}^{T}\right)\right)$ exists and does not depend on $t$

$\mathrm{A}_{4}$ :

$$
\lim _{n \rightarrow \infty} \frac{1}{n} \sum_{t=1}^{n} E_{\theta^{0}}\left(\frac{\partial e_{t}^{T}(\theta)}{\partial \theta_{i}} \Sigma^{-1} \frac{\partial e_{t}(\theta)}{\partial \theta_{j}}\right)=V_{i j},
$$

for $i, j=1, \ldots, m$, where the matrix $V=\left(V_{i j}\right)_{1 \leq i, j \leq m}$ is strictly positive definite; 
$\mathbf{A}_{5}$ :

$\mathbf{A}_{6}$ :

$$
\frac{1}{n^{2}} \sum_{d=1}^{n-1} \sum_{t=1}^{n-d} \sum_{k=1}^{t-1}\left\|\psi_{t i k}\right\|_{F}\left\|\psi_{t+d, j, k+d}\right\|_{F}=O\left(\frac{1}{n}\right), \quad i, j=1, \ldots, m
$$

$$
\begin{aligned}
\frac{1}{n^{2}} \sum_{d=1}^{n-1} \sum_{t=1}^{n-d}\left[\sum_{k=1}^{t-1} M_{t 0 k k}^{j i T} \Xi(\Sigma) M_{t d k k}^{i j}+\right. & \sum_{k_{1}=1}^{t-1} \sum_{k_{2}=1}^{t-1} M_{t 0 k_{2} k_{1}}^{j i T} K_{r, r}(\Sigma \otimes \Sigma) M_{t d k_{1} k_{2}}^{i j} \\
& \left.+\sum_{k_{1}=1}^{t-1} \sum_{k_{2}=1}^{t-1} M_{t 0 k_{2} k_{1}}^{j i T}(\Sigma \otimes \Sigma) M_{t d k_{2} k_{1}}^{i j}\right]=O\left(\frac{1}{n}\right),
\end{aligned}
$$

with $\Xi(\Sigma)=\kappa-\operatorname{vec}(\Sigma)$. $\operatorname{vec}(\Sigma)^{T}-(\Sigma \otimes \Sigma)-K_{r, r}(\Sigma \otimes \Sigma)$, and, for $k^{\prime}, k^{\prime \prime}=k, k_{1}, k_{2}$, $M_{t f k^{\prime} k^{\prime \prime}}^{i j}=\operatorname{vec}\left(\psi_{t+f, i, k^{\prime}+f}^{T} \Sigma^{-1} \psi_{t+f, j, k^{\prime \prime}+f}\right), f=0, d, i, j=1, \ldots, m$.

Theorem 3.1. Under the assumptions $\mathbf{A}_{1}-\mathbf{A}_{6}$, the estimator $\widehat{\theta}_{n}$ introduced in Section 2.2 converges almost surely to the true value $\theta^{0}$ and $n^{1 / 2}\left(\widehat{\theta}_{n}-\theta^{0}\right) \stackrel{L}{\rightarrow} N\left(0, V^{-1}\right)$ when $n \rightarrow \infty$.

Except in one aspect, Theorem 3.1 is a corollary of Alj et al. (2017, Theorem 2), where it is deduced from Alj et al. (2017, Theorem 1), which is itself a consequence of Klimko and Nelson's (1978) general theorems for strong consistency and asymptotic normality. The exception is that Alj et al. (2017) suppose that $\epsilon_{t}$ has finite eighthorder moments. That assumption could be weakened to moments of order $4+\delta, \delta>0$, using Theorem 2.3 in Azrak and Mélard (2020); see also Alj et al. (2020). Moreover, for a homoscedastic model such as ours, the existence of fourth-order moments as in $\mathbf{A}_{3}$ above is even enough.

Remark 3.1. The assumptions $\mathbf{A}_{1}, \mathbf{A}_{2}, \mathbf{A}_{4}, \mathbf{A}_{5}$, and $\mathbf{A}_{6}$, correspond, respectively, to $\mathbf{H}_{2.1}, \mathbf{H}_{2.2}, \mathbf{H}_{2.6}, \mathbf{H}_{2.7}(A)$, and $\mathbf{H}_{2.7}(B)$ in Alj et al. (2017). Note that in $\mathbf{A}_{2}$ we have omitted the polynomial in $\nu$ in the right-hand side of the first four inequalities of $\mathbf{H}_{2.2}$ because it is not necessary. The last two inequalities in $\mathbf{A}_{2}$ were missing in $\mathbf{H}_{2.2}$. They can be used to prove that the process $x_{t}$ has fourth-order moments; see Alj et al. (2020) - a referee brought this requirement to our attention. The other assumptions $\mathbf{H}_{2.3}, \mathbf{H}_{2.4}(c), \mathbf{H}_{2.5}$ are not needed here. Alj et al. (2017) had a further assumption $\mathbf{H}_{2.8}$ about the existence of a definite positive matrix $W$ which is the outer product of gradient that should appear in the asymptotic covariance matrix as a socalled sandwich expression $V^{-1} W V^{-1}$. As justified by Alj et al. (2020, Section 3.4), $W=V$ in the present homoscedastic context, so $\mathbf{H}_{2.8}$ brings nothing new. For ARMA models, i.e. when $r=1$, the Frobenius norms are replaced by absolute values in $\mathbf{A}_{2}$ and $\mathbf{A}_{5}, \kappa$ is the fourth-order cumulant of $\epsilon_{t}$ in $\mathbf{A}_{4}$, and $\mathbf{A}_{6}$ takes a much simpler form (see the Supplementary Material, where a full proof is given). 
Remark 3.2. A referee noticed that a key condition for strong consistency is given in Klimko and Nelson (1978, Theorem 2.1 (iii)) but cannot be found in Alj et al. (2017). This is probably because the proof was omitted by Alj et al. (201\%, Theorem 1) as it was too similar to that of Azrak and Mélard (2006, Theorem 1), the only difference being that $\alpha_{t}(\theta)$ is a quadratic form in the former and a square in the latter. A strong law of large numbers for martingale sequences is used for that purpose in Azrak and Mélard (2006). Since the assumptions in Alj et al. (2017, Theorem 2) are intended to prove the assumptions of Alj et al. (2017, Theorem 1), strong consistency is guaranteed. See the Supplementary Material for the scalar ARMA model.

Remark 3.3. We use the conditional Gaussian likelihood function, not the exact Gaussian likelihood. In the stationary case, they are asymptotically equivalent. We conjecture that this equivalence should be valid for time-dependent models, under suitable assumptions, but that remains to be proven.

\subsection{Some properties of polynomials}

Before considering VARMA models, let us state a few lemmas, including one that formalizes and extends what was done in Alj et al. (2017, Appendix S1, Eq. (A.19)).

Lemma 3.1. [Ruiz, 1996] For all integers $n \geq 0$ and for $j$ integer such that $1 \leq j \leq n$

$$
\sum_{i=0}^{n}(-1)^{i}\left(\begin{array}{c}
n \\
i
\end{array}\right)(x-i)^{n-j}=0 .
$$

Corollary 3.1. For all integers $n \geq 0$ and for any polynomial $P_{n-1}(x)$ of degree at most $n-1$, for all real numbers $x$

$$
\sum_{i=0}^{n}(-1)^{i}\left(\begin{array}{c}
n \\
i
\end{array}\right) P_{n-1}(x)=0 .
$$

Lemma 3.2. For all real numbers $x$ such that $|x|<1$, and any polynomial $P_{m}(k)$ of degree $m \geq 0$ in $k$,

$$
\sum_{k=0}^{\infty} P_{m}(k) x^{k}
$$

is bounded by a constant multiplied by $1 /(1-x)^{m+1}$.

Proof of Lemma 3.2. This is trivial for $m=0$, using the sum of the geometric series. For $m \geq 1$, we denote $P_{m}(k)=\sum_{j=0}^{m} c_{j} k^{j}$ and consider

$$
(1-x)^{m+1}=\sum_{i=0}^{m+1}(-1)^{i}\left(\begin{array}{c}
m+1 \\
i
\end{array}\right) x^{i}
$$


and multiply it by $(3.23)$ and regroup the terms in $x^{k}$ for $k \geq m+1$. The coefficient of $x^{k}$ is

$$
\begin{aligned}
& \sum_{i=0}^{m+1}(-1)^{i}\left(\begin{array}{c}
m+1 \\
i
\end{array}\right) \sum_{j=0}^{m}(-1)^{j}\left(\begin{array}{c}
m \\
j
\end{array}\right) c_{j} k^{m-j} i^{j} \\
= & \sum_{j=0}^{m}(-1)^{j}\left(\begin{array}{c}
m \\
j
\end{array}\right) c_{j} \sum_{i=0}^{m+1}(-1)^{i}\left(\begin{array}{c}
m+1 \\
i
\end{array}\right) i^{j} .
\end{aligned}
$$

Since $j \leq m$ and using Corollary 3.1, the internal sum

$$
\sum_{i=0}^{m+1}(-1)^{i}\left(\begin{array}{c}
m+1 \\
i
\end{array}\right) i^{j}
$$

is identically zero. Consequently, all terms for $k \geq m+1$ cancel out in the product of 3.23 by 3.24. There remains a finite number of terms whose sum is a finite constant $K$. Hence, 3.23 is bounded by $K /(1-x)^{m+1}$.

\section{Main result}

Starting in this section, we consider the standard VARMA model, defined by (2.3), as a special case of (2.2). We now suppose constant matrices with respect to time, hence $A_{t}(\theta)=A(\theta)$, and $B_{t}(\theta)=B(\theta)$, for all $t$ and $\theta$, where, to simplify the presentation, $\theta$ is composed of the elements of the coefficients of the model, more precisely

$$
\theta^{0}=\left(\operatorname{vec}\left(A_{1}\right)^{T}, \ldots, \operatorname{vec}\left(A_{p}\right)^{T}, \operatorname{vec}\left(B_{1}\right)^{T}, \ldots, \operatorname{vec}\left(B_{q}\right)^{T}\right)^{T} .
$$

In principle, the vector $\theta$ can be a subset of those elements, but that would complicate the notations. The elements of the invertible matrix $\Sigma$ are treated as nuisance parameters.

\subsection{Assumptions for the VARMA process}

We assume the following for the VARMA process:

$\mathbf{B}_{1}$ : for all $t$, the errors $\epsilon_{t}$ are i.i.d. random vectors with covariance matrix $\Sigma$ and $\kappa=E\left(\operatorname{vec}\left(\epsilon_{t} \epsilon_{t}^{T}\right) \operatorname{vec}\left(\epsilon_{t} \epsilon_{t}^{T}\right)^{T}\right)$ exists (and does not depend on $t$ );

$\mathbf{B}_{2}$ : the process is causal, i.e. the matrix polynomial $A(z)=I_{r}-\sum_{i=1}^{p} A_{i} z^{i}$ has all its eigenvalues (the roots of the determinantal equation $\operatorname{det}(A(z))=0$ ) outside of the unit circle of the complex disc; 
$\mathbf{B}_{3}$ : the process is invertible, i.e. the matrix polynomial $B(z)=I_{r}+\sum_{j=1}^{q} B_{j} z^{j}$ has all its eigenvalues (the roots of the determinantal equation $\operatorname{det}(B(z))=0$ ) outside of the unit circle of the complex disc;

$\mathbf{B}_{4}$ : the process is identifiable, i.e. the two identifiability conditions are satisfied: $\left(\mathbf{B}_{4} i\right)$ left coprimeness of $A(z)$ and $B(z)$, and $\left(\mathbf{B}_{4} i i\right)$ with an additional condition that the matrix obtained by the juxtaposition of $A_{p}$ and $B_{q}$ has full rank.

We will give a sufficient condition of identifiability in Section 4.7 .

Remark 4.1. For an ARMA model, i.e. with $r=1$, the first assumption is: $\left(\mathbf{B}_{1}\right)$ the errors $\epsilon_{t}$ are i.i.d. random variables with variance $\sigma^{2}$ and a fourth-order cumulant $\kappa$. The causality $\left(\mathbf{B}_{2}\right)$ and invertibility $\left(\mathbf{B}_{3}\right)$ conditions are expressed by the roots of the scalar polynomials $A(z)=1-\sum_{i=1}^{p} A_{i} z^{i}$ and $B(z)=1+\sum_{j=1}^{q} B_{j} z^{j}$, respectively, being all outside of the unit circle of the complex disc. The identifiability condition $\left(\mathbf{B}_{4}\right)$ is simpler: the roots of $A(z)$ are all different from those of $B(z)$.

Remark 4.2. We have mentioned structural VARMA models in the introduction. There are several kinds of structural VARMA models, but they are often used in attempts to express a particular theory, for instance, an economic theory. Some of them do not even satisfy the invertibility condition-see Gouriéroux et al. (2020). A first kind is when (2.3) is replaced by the equation

$$
A_{0}(\theta) x_{t}=\sum_{i=1}^{p} A_{i}(\theta) x_{t-i}+B_{0}(\theta) e_{t}(\theta)+\sum_{j=1}^{q} B_{j}(\theta) e_{t-j}(\theta)
$$

thus with extra matrices $A_{0}(\theta)$ and $B_{0}(\theta)$ instead of an identity matrix, with some restrictions, of course. This is the case of the echelon form and the scalar multiple components that will be mentioned later in Remark 4.7 and also of Boubacar Maïnassara and Francq (2011) in the more general case of non-independent errors. Another kind of structural model is when there are relations, not necessarily linearly, among the coefficients, as in the seasonal VARMA models; see Reinsel (1997, Section 6.5) and Yozgatligil and Wei (2009). Reinsel (1997, Section 5.2) and Lütkepohl (2005, Section 12.2.4) deal with linear restrictions in VARMA models. Both Hannan and Deistler (2012, Section 4.3) and Kohn (1979) consider estimation of VARMAX models (i.e. VARMA models with additional exogenous variables) under constraints among the parameters. Hannan and Deistler (2012, Section 2.7) present several sufficient conditions results for the identifiability of structural VARMAX models that are not as strict as for the fully-parametrized VARMA models defined above.

Alj et al. (2017) do not consider a time-dependent generalization of (4.2) with matrices $A_{0}(\theta)$ and $B_{0}(\theta)$ different from $I_{r}$ so, we cannot formally refer to the assumptions in Section 3.3. There can also be dependencies among the coefficients in the case of structural VARMA models, and this would complicate the presentation, particularly in Section 4.7. Nevertheless, there is no doubt that, at least in a large number of cases 
(but excluding non-invertible models), these structural models can be considered, with an appropriate change of $\mathbf{B}_{4}$.

Remark 4.3. As mentioned in the introduction, this paper is also applicable to scalar ARMA models. Note, however, that only the existence of the variance is needed by Yao and Brockwell (2006) in that case, not fourth-order moments. The latter assumption is also made by Ling and McAleer (2003) but in the wider context of VARMA-ARCH models. The assumption of a Gaussian process is made by Rissanen and Caines (1979). For models with weak white noise errors, moments of the order $4+\delta, \delta>0$ are assumed by Boubacar Maïnassara and Francq (2011). Most authors suppose i.i.d. innovations, except Hannan and Deistler (2012, Section 4.1) (with different set-ups), Kohn (1979) (stationary square martingale differences), and, of course, Boubacar Maïnassara and Francq (2011) (stationary and ergodic non-autocorrelated errors with a mixing condition). Unlike in e.g. Boubacar Maïnassara and Saussereau (2018) and Dufour and Pelletier (2019), independence of the errors is a requirement for the tdVARMA models in Alj et al. (2017). Several authors consider an open parameter space $\Theta$ like we do, with the exceptions of Kohn (1978, 1979), Boubacar Maïnassara and Francq (2011), and Ling and McAleer (2003).

\subsection{Main theorem}

Theorem 4.1. For any $\operatorname{VARMA}(p, q)$ model defined by (2.3) and satisfying the assumptions $\mathbf{B}_{1}-\mathbf{B}_{4}$, the Gaussian pseudo-likelihood estimator $\widehat{\theta}_{n}$ is almost surely convergent to the true value $\theta^{0}$, when $n \rightarrow \infty$, and is also asymptotically normal with covariance matrix $V^{-1}$, where $V$ is the Fisher information matrix, which is invertible, i.e. $n^{1 / 2}\left(\widehat{\theta}_{n}-\theta^{0}\right) \stackrel{L}{\rightarrow} N\left(0, V^{-1}\right)$.

The proof consists in checking the assumptions $\mathbf{A}_{1}-\mathbf{A}_{6}$ of Theorem 3.1 as in Alj et al. (2017, Theorem 2) particularized in Section 3.4 for i.i.d. errors and an identity matrix $g_{t}$. To make the proof possible for models with $p+q>1$, we will first consider the $\operatorname{VAR}(1)$ representation of Section 3.1.

\subsection{Application of the $V A R(1)$ representation}

From now on, we omit the useless subscripts $t$, so we write e.g. $\Psi_{i k}(\theta)$ for $\Psi_{t i k}(\theta)$, or $\psi_{i k}(\theta)$ for $\psi_{t i k}(\theta)$. So a product like $\left(\prod_{j=0}^{s-1} \phi_{t-j}^{0}\right)$ in 3.16 is simply a power $\left(\phi^{0}\right)^{s}$, and, similarly, $\left(\prod_{\ell=0}^{k-s-1} J \phi_{t-\ell}(\theta)\right)$ is equal to $(J \phi(\theta))^{k-s}$. For the purpose of the next section, we need the Frobenius norm of $\Psi_{i k}(\theta)$ and those corresponding to second and third derivatives for VARMA models.

We will first evaluate the products involved in $(3.16)$ as well as in $\Psi_{i k}(\theta)$ defined in (3.22). For $k>0$, let us consider the $k$-th power $\phi^{k}(\theta)$ of $\phi(\theta)$, defined by 3.2 . This has the following form

$$
\phi^{k}(\theta)=\left(\begin{array}{cc}
A^{k}(\theta) & D_{k}(\theta) \\
O_{q, p} & C^{k}
\end{array}\right),
$$


where, omitting the arguments $\theta$ of $A(\theta)$ and $\tilde{B}(\theta)$ for simplicity in the right-hand sides, the upper right block is

$$
\begin{aligned}
D_{k}(\theta) & =A^{k-1} \tilde{B}+A^{k-2} \tilde{B} C+\ldots+A^{k-q} \tilde{B} C^{q-1} \\
& =A^{k-q}\left(A^{q-1} \tilde{B}+A^{q-2} \tilde{B} C+\ldots+\tilde{B} C^{q-1}\right)
\end{aligned}
$$

since $C^{k}$ is a lower-shift block $q \times q$ matrix with $I_{r}$ in block position $(k+1,1)$, hence $C^{k}=0_{r}$ for $k \geq q$. Note that 4.3 and (4.4) are a special case of Francq and Zakoïan 2001, Eq. (4)). Let us define $D_{k}^{0}=D_{k}\left(\theta^{0}\right)$.

Similarly, given the structure of $J \phi(\theta)$ in 3.11 , we have for $(J \phi(\theta))^{k}$ the following form

$$
(J \phi(\theta))^{k}=\left(\begin{array}{cc}
\tilde{C}^{k} & O_{p, q} \\
\tilde{D}_{k}(\theta) & B^{k}(\theta)
\end{array}\right),
$$

where the lower left block (omitting again dependency on $\theta$ for $\tilde{A}$ and $B$ ) is given by

$$
\begin{aligned}
\tilde{D}_{k}(\theta) & =B^{k-1} \tilde{A}+B^{k-2} \tilde{A} \tilde{C}+\ldots+B^{k-p} \tilde{A} \tilde{C}^{p-1} \\
& =B^{k-p}\left(B^{p-1} \tilde{A}+B^{p-2} \tilde{A} \tilde{C}+\ldots+\tilde{A} \tilde{C}^{p-1}\right)
\end{aligned}
$$

because $\tilde{C}^{k}=0_{r}$ for $k \geq p, \tilde{C}^{k}$ is a lower-shift block $p \times p$ matrix with $I_{r}$ in block position $(k+1,1)$. Let us define $\tilde{D}_{k}^{0}=\tilde{D}_{k}\left(\theta^{0}\right)$.

\subsection{Verification of $\mathbf{A}_{2}$}

Lemma 4.1. Under the assumptions $\mathbf{B}_{1}-\mathbf{B}_{4}$, Assumption $\mathbf{A}_{2}$ is verified.

This assumption bears on the coefficients of the pure moving average representation of the derivatives of the residuals with respect to the parameters. It is easier to check the assumption for the $\Psi_{i k}$ than for the $\psi_{i k}$ based on 3.22 .

We need to examine the first three derivatives of (3.16). As before, let us consider only the first derivative with respect to $\theta_{i}, i=1, \ldots, m$, since it will be very similar for the other two derivatives.

Note first that the derivative with respect to $\theta_{i}, i=1, \ldots, m$, of $(J \phi(\theta))^{k-s}$ is given by

$$
\frac{\partial(J \phi(\theta))^{k-s}}{\partial \theta_{i}}=\sum_{\ell=1}^{k-s}(J \phi(\theta))^{\ell-1} \frac{\partial J \phi(\theta)}{\partial \theta_{i}}(J \phi(\theta))^{k-s-\ell} .
$$

Lemma 4.2. Suppose that the largest eigenvalue in modulus of the two reciprocal matrix polynomials $A^{*}(z)$ and $B^{*}(z)$ is smaller than $\Phi<1$, then there exists $\tilde{k}$ such that, for any integer $k>\tilde{k}$, we have

$$
\text { (a) }\left\|\left(\phi^{0}\right)^{k}\right\|_{F}<M \Phi^{k} \quad \text { and }(b)\left\|\left\{\partial(J \phi(\theta))^{k} / \partial \theta_{i}\right\}_{\theta=\theta^{0}}\right\|_{F}<M k \Phi^{k}
$$


where $M$ is a positive constant.

Proof of Lemma 4.2.

Note that $\|K\|_{F}=2$ and $\left\|\left(\partial J \phi(\theta) / \partial \theta_{i}\right)_{\theta=\theta^{0}}\right\|_{F}$ is a finite integer. By simple calculations based on (4.3), $\left\|\left(\phi^{0}\right)^{k}\right\|_{F}^{2}=\operatorname{tr}\left(\left(A^{0}\right)^{k}\left(A^{0 T}\right)^{k}\right)+\operatorname{tr}\left(C^{k} C^{k T}\right)+\operatorname{tr}\left(D_{k}^{0} D_{k}^{0 T}\right)$. Since all the matrices involved in (4.4) have a finite Frobenius norm and the number of terms $q$ is finite, $\left\|\left(\phi^{0}\right)^{k}\right\|_{F}$ is bounded by $M\left\|\left(A^{0}\right)^{k}\right\|_{F}$, where $M$ is a positive constant. But the causality assumption implies that the eigenvalues of the companion matrix $A^{0}$ have modulus smaller than $\Phi<1$ so that $\left\|\left(A^{0}\right)^{k}\right\|_{F}<\Phi^{k}$, proving part (a) of the assertion. The proof for part (b) of the assertion is partially similar, but is based on (4.7), 4.5), and (4.6). Instead of causality, we make use of the invertibility assumption, which implies that the eigenvalues of the companion matrix $B^{0}$ have modulus smaller than $\Phi<1$, so that $\left\|\left(B^{0}\right)^{k}\right\|_{F}<\Phi^{k}$. The factor $k$ in the right-hand side of 4.8)(b) comes from the number of terms in the derivative (4.7).

Remark 4.4. $\left\|\left(\partial J \phi(\theta) / \partial \theta_{i}\right)_{\theta=\theta^{0}}\right\|_{F}$ is even equal to 1 if we assume (4.1). With other realistic parametrizations, we will not have 1 but another finite positive integer.

Proof of Lemma 4.1. According to Assumption $\mathbf{A}_{2}$ in Section 4.3, we need to show that there exists a positive constant $N_{1}$ and $0<\Phi<1$ such that, for $\nu=1, \ldots, t-1$, $\sum_{k=\nu}^{t-1}\left\|\Psi_{i k}\right\|_{F}^{2}<N_{1} \Phi^{\nu-1}$. Using the properties of the Frobenius norm, 3.16) and Lemma 4.2 imply that

$$
\begin{aligned}
\left\|\Psi_{i k}\right\|_{F} & \leq 2 M^{2} \sum_{s=0}^{k}\left(\sum_{\ell=1}^{k-s} \Phi^{k-s-1}\right) \Phi^{s} \\
& =2 M^{2} \sum_{s=0}^{k}(k-s) \Phi^{k-1} \leq M^{2} k(k+1) \Phi^{k-1},
\end{aligned}
$$

for $i=1, \ldots, m$. As a consequence

$$
\begin{aligned}
\sum_{k=\nu}^{t-1}\left\|\Psi_{i k}\right\|_{F}^{2} & \leq M^{4} \sum_{k=\nu}^{t-1}(k(k+1))^{2} \Phi^{2(k-1)} \\
& \leq M^{4} \Phi^{2(\nu-1)} \sum_{k=\nu}^{\infty}(k(k+1))^{2} \Phi^{2(k-\nu)}
\end{aligned}
$$

Using Lemma 3.2, this can be bounded by $M^{4} \Phi^{2(\nu-1)} /\left(1-\Phi^{2}\right)^{5}$ because of the polynomial in $k$ of degree 4 in the sum 4.10 . That proves the first part of $\mathbf{A}_{2}$. The second part is about a similar property for $\sum_{k=\nu}^{t-1}\left\|\Psi_{i k}\right\|_{F}^{4}$, with $i=1, \ldots, m$, for which the proof is similar, with a polynomial in $k$ of degree 8 , and is therefore omitted. 
The next three parts involve the coefficients $\Psi_{i j k}$ and $\Psi_{i j l k}, i, j, l=1, \ldots, m$, related to second- and third-order derivatives of $e_{t}(\theta)$, and can be handled by similar arguments. The last two inequalities are direct consequences of (3.16), Lemma 4.2, and the convergence of a geometric series.

\subsection{Verification of $\mathbf{A}_{5}$}

Lemma 4.3. Under the assumptions $\mathbf{B}_{1}-\mathbf{B}_{4}$, Assumption $\mathbf{A}_{5}$ is verified.

Proof of Lemma 4.3. It is again easier to check the assumption for the $\Psi_{i k}$ instead of for the $\psi_{i k}$. According to Assumption $\mathbf{A}_{5}$, we should have

$$
\frac{1}{n^{2}} \sum_{d=1}^{n-1} \sum_{t=1}^{n-d} \sum_{k=1}^{t-1}\left\|\Psi_{i k}\right\|_{F}\left\|\Psi_{j, k+d}\right\|_{F}=O\left(\frac{1}{n}\right) .
$$

We can permute the sums as follows

$$
\sum_{d=1}^{n-1} \sum_{t=1}^{n-d} \sum_{k=1}^{t-1}(.)=\sum_{d=1}^{n-1} \sum_{k=1}^{n-d-1} \sum_{t=k+1}^{n-d}(.)
$$

Hence, given that the general term does not depend on $t$, the triple sum in 4.11) can be bounded from above by

$$
\begin{aligned}
& \sum_{d=1}^{n-1} \sum_{k=1}^{n-d-1}(n-d-k)\left\|\Psi_{i k}\right\|_{F}\left\|\Psi_{j, k+d}\right\|_{F} \\
\leq & M^{4} \sum_{d=1}^{n-1} \sum_{k=1}^{n-d-1}(n-d-k) k(k+1)(k+d)(k+d+1) \Phi^{2 k+d-2} \\
\leq & n M^{4} \sum_{d=1}^{n-1} \Phi^{d-2} \sum_{k=1}^{\infty}(k+d+1)^{4} \Phi^{2 k}
\end{aligned}
$$

because of 4.9. Using Lemma 3.2, an upper bound of the general term of the internal sum is a polynomial $P_{4}(d)$ in $d$ of degree 4 divided by $\left(1-\Phi^{2}\right)^{5}$. We have, thus, $\left\{n M^{4} /\left(1-\Phi^{2}\right)^{5}\right\} \sum_{d=1}^{n-1} P_{4}(d) \Phi^{d-2}$. Using Lemma 3.2 again on the sum over $d$ extended to $\infty$, we have another upper bound $C \Phi^{-2} /(1-\Phi)^{5}$ for the sum, where $C$ is a constant, hence $\left\{n M^{4} C \Phi^{2} /\left\{\left(1-\Phi^{2}\right)^{5}(1-\Phi)^{5}\right\}\right.$. If we multiply this by $1 / n^{2}$ as in (4.11), we obtain $O(1 / n)$, as requested.

\subsection{Verification of $\mathbf{A}_{6}$}

Lemma 4.4. Under the assumptions $\mathbf{B}_{1}-\mathbf{B}_{4}$, Assumption $\mathbf{A}_{6}$ is verified. 
Proof of Lemma 4.4. Here, working with the big VAR(1) model, we would face the problem that the covariance matrix of the $E_{t}^{0}$ is singular. For that reason, we will, instead, transform the $\Psi_{i k}$ into the $\psi_{i k}$. According to Assumption $\mathbf{A}_{6}$, taking care that no term depends on $t$, and therefore omitting the subscripts $t$ everywhere, we should have

$$
\begin{aligned}
\frac{1}{n^{2}} \sum_{d=1}^{n-1} \sum_{t=1}^{n-d}\left[\sum_{k=1}^{t-1} M_{0 k k}^{j i T} \Xi(\Sigma) M_{d k k}^{i j}\right. & +\sum_{k_{1}=1}^{t-1} \sum_{k_{2}=1}^{t-1} M_{0 k_{2} k_{1}}^{j i T} K_{r, r}(\Sigma \otimes \Sigma) M_{d k_{1} k_{2}}^{i j} \\
& \left.+\sum_{k_{1}=1}^{t-1} \sum_{k_{2}=1}^{t-1} M_{0 k_{2} k_{1}}^{j i T}(\Sigma \otimes \Sigma) M_{d k_{2} k_{1}}^{i j}\right]=O\left(\frac{1}{n}\right)
\end{aligned}
$$

with $\Xi(\Sigma)=\kappa-\operatorname{vec}(\Sigma) \cdot \operatorname{vec}(\Sigma)^{T}-(\Sigma \otimes \Sigma)-K_{r, r}(\Sigma \otimes \Sigma)$, and, for $k^{\prime}, k^{\prime \prime}=k, k_{1}, k_{2}$, $N_{f k^{\prime} k^{\prime \prime}}^{i j}=\psi_{i, k^{\prime}+f}^{T} \Sigma^{-1} \psi_{j, k^{\prime \prime}+f}$ and $M_{f k^{\prime} k^{\prime \prime}}^{i j}=\operatorname{vec}\left(N_{f k^{\prime} k^{\prime \prime}}^{i j}\right), f=0, d$. As in the previous subsection, the order of summation can be changed. We use 4.12) again for the first term, but for the two other terms, we use instead

$$
\sum_{d=1}^{n-1} \sum_{t=1}^{n-d} \sum_{k_{1}=1}^{t-1} \sum_{k_{2}=1}^{t-1}(.) \leq \sum_{d=1}^{n-1} \sum_{k_{1}=1}^{n-d-1} \sum_{k_{2}=1}^{n-d-1} \sum_{t=\min \left(k_{1}, k_{2}\right)+1}^{n-d}(.)
$$

We will evaluate the Frobenius norm of the left-hand side of 4.13 and show that it is $O(1 / n)$. First $\left\|M_{f k^{\prime} k^{\prime \prime}}^{i j}\right\|_{F}=\left\|N_{f k^{\prime} k^{\prime \prime}}^{i j}\right\|_{F}$. Then

$$
\left\|N_{f k^{\prime} k^{\prime \prime}}^{i j}\right\|_{F} \leq\left\|\psi_{i, k^{\prime}+f}^{T}\right\|_{F}\left\|\Sigma^{-1}\right\|_{F}\left\|\psi_{j, k^{\prime \prime}+f}\right\|_{F} .
$$

Given (3.22) and the fact that $\left\|I_{r}\right\|_{F}=\sqrt{r}$, we have $\left\|\psi_{i, k^{\prime}+f}\right\|_{F} \leq r\left\|\Psi_{i, k^{\prime}+f}\right\|_{F}$, hence $\left\|M_{f k^{\prime} k^{\prime \prime}}^{i j}\right\|_{F} \leq r^{2}\left\|\Sigma^{-1}\right\|_{F}\left\|\Psi_{i, k^{\prime}+f}\right\|_{F}\left\|\Psi_{j, k^{\prime \prime}+f}\right\|_{F}$. Using (4.9), this is bounded by $r^{2} M^{4}\left\|\Sigma^{-1}\right\|_{F}\left(k^{\prime}+f+1\right)^{2}\left(k^{\prime \prime}+f+1\right)^{2} \Phi^{k^{\prime}+k^{\prime \prime}+2 f-2}$. The matrices in the centre of the terms of the three sums in (4.13) have a finite Frobenius norm and they do not depend on the summation indices. We denote $S=\max \left(\|\Xi(\Sigma)\|_{F},\left\|K_{r, r}(\Sigma \otimes \Sigma)\right\|_{F},\|\Sigma \otimes \Sigma\|_{F}\right)$. Since the last two terms of 4.13) can be grouped and, after using 4.14, the sum over $t$ is a constant bounded by $(n-d) \leq n$, the left-hand side of 4.13 is bounded by

$$
\begin{aligned}
& \frac{S r^{4} M^{8}\left\|\Sigma^{-1}\right\|_{F}^{2}}{n^{2}}\left(\sum_{d=1}^{n-1} \sum_{k=1}^{n-d-1}(n-d)(k+1)^{4}(k+d+1)^{4} \Phi^{2(4 k+2 d-4)}\right. \\
& \left.+2 \sum_{d=1}^{n-1} \sum_{k_{1}=1}^{n-d-1} \sum_{k_{2}=1}^{n-d-1}(n-d)\left(k_{1}+d+1\right)^{4}\left(k_{2}+d+1\right)^{4} \Phi^{2\left(2 k_{1}+2 k_{2}+2 d-4\right)}\right) .
\end{aligned}
$$


For the first term, we proceed as in Section 4.4, except that the polynomial has degree 8 here, instead of 4 , to obtain $O(1 / n)$. For the second term, the last two sums can be factored and handled in the same way with polynomials of degree 4 each, giving again $O(1 / n)$.

\subsection{Verification of $\mathbf{A}_{4}$}

Lemma 4.5. Under the assumptions for a VARMA model including identifiability, in Assumptions $\mathbf{B}_{4} i$ and $\mathbf{B}_{4}$ ii, Assumption $\mathbf{A}_{4}$ is verified.

This section is not necessary for ARMA models - see Whittle (1953a). As a matter of fact, we will not check $\mathbf{A}_{4}$; we will, however, directly check the existence and invertibility of the Fisher information matrix $V$. Strangely enough, this verification is not provided in Hannan and Deistler s proof (2012, Chapter 4).

For VARMA models, the expression of $V$ was first investigated by Newton (1978), who also provides algorithms. It is based on the expression of the asymptotic covariance matrix for Gaussian maximum likelihood estimators by Whittle (1953b) and the expression of the spectral density of a VARMA process by Hannan (1970). But Newton (1978) does not discuss the invertibility of $V$.

In Section 2.1 and in Section 4.1, we have mentioned a double identifiability assumption $\mathbf{B}_{4}$ in addition to the causality and invertibility conditions $\mathbf{B}_{2}-\mathbf{B}_{3}$, which are maintained through the whole paper. Identifiability means that the structure defined by $A(z)$ and $B(z)$, of respective degrees $p$ and $q$, is unique in the sense that for a given infinite moving average representation defined by $\psi(z)=A^{-1}(z) B(z)$, there are no other $r \times r$ matrices of polynomials $A_{1}(z)$ and $B_{1}(z)$, with the same respective degrees $p$ and $q$, such that $A_{1}^{-1}(z) B_{1}(z)=\psi(z)$. Clearly, if $A(z)$ and $B(z)$ can be factorized as $F(z) A_{1}(z)$ and $F(z) B_{1}(z)$, of course with an invertible $r \times r$ matrix $F$, then $A^{-1}(z) B(z)=A_{1}^{-1}(z) B_{1}(z)$. In general, $A_{1}(z)$ and $B_{1}(z)$ will have smaller degrees than $A(z)$ and $B(z)$, respectively. This is solved by the condition $\mathbf{B}_{4} \mathrm{i}$, that $A(z)$ and $B(z)$ are left-coprime. In addition to $\mathbf{B}_{4} \mathbf{i}$, Hannan (1969) supposes the condition $\mathbf{B}_{4}$ ii, that the matrix obtained by the juxtaposition of $A_{p}$ and $B_{q}$ has full rank. Note that Hannan (1969) even states $\mathbf{B}_{4} \mathrm{i}-\mathbf{B}_{4}$ ii as a necessary and sufficient condition that is not retained in subsequent publications, such as Hannan and Deistler (2012). Several book authors mention $\mathbf{B}_{4}$ ii, including Reinsel (1997, Section 2.3.4), who called it block identifiability, and Tsay (2014, Section 3.5). Several authors, including Hallin et al. (2020), assume that $\operatorname{det}\left(A_{p}\right) \neq 0 \neq \operatorname{det}\left(B_{q}\right)$. This, together with $\mathbf{B}_{4} \mathrm{i}$, is a sufficient but not a necessary condition for $\mathbf{B}_{4}$ ii. 
We now give the proof based on Ling and McAleer (2003, Lemma 4.2) given by them for VARMA-GARCH models, with several minor corrections. After that, we will discuss the conditions.

Proof of Lemma 4.5. We define the derivative of an $r \times 1$ vector with respect to an $1 \times m$ row vector as an $r \times m$ matrix. Taking the derivative of 2.3 with respect to $\theta^{T}$, we have

$$
\frac{\partial\left[e_{t}(\theta)+\sum_{j=1}^{q} B_{j}(\theta) e_{t-j}(\theta)\right]}{\partial \theta^{T}}=\frac{\partial\left[x_{t}-\sum_{i=1}^{p} A_{i}(\theta) x_{t-i}\right]}{\partial \theta^{T}} .
$$

Using standard rules for derivatives of vectors, e.g. Lütkepohl (2005, Appendix A), the right-hand side of 4.15 is equal to

$$
-\sum_{i=1}^{p}\left(x_{t-i}^{T} \otimes I_{r}\right) \frac{\partial \operatorname{vec} A_{i}(\theta)}{\partial \theta^{T}}
$$

while the left-hand side of 4.15 is equal to

$$
\frac{\partial e_{t}(\theta)}{\partial \theta^{T}}+\sum_{j=1}^{q}\left[B_{j}(\theta) \frac{\partial e_{t-j}(\theta)}{\partial \theta^{T}}+\left(e_{t-j}^{T}(\theta) \otimes I_{r}\right) \frac{\partial \operatorname{vec} B_{j}(\theta)}{\partial \theta^{T}}\right]
$$

Hence 4.15) implies

$$
\frac{\partial e_{t}(\theta)}{\partial \theta^{T}}+\sum_{j=1}^{q} B_{j}(\theta) \frac{\partial e_{t-j}(\theta)}{\partial \theta^{T}}=-\sum_{i=1}^{p}\left(x_{t-i}^{T} \otimes I_{r}\right) \frac{\partial \operatorname{vec} A_{i}(\theta)}{\partial \theta^{T}}-\sum_{j=1}^{q}\left(e_{t-j}^{T}(\theta) \otimes I_{r}\right) \frac{\partial \operatorname{vec} B_{j}(\theta)}{\partial \theta^{T}}
$$

which can be written as follows for $\theta=\theta^{0}$, using the lag operator $L$, the $r(p+$ $q) \times 1$ vector $X_{t-1}(\theta)=\left(x_{t-1}^{T}, \ldots, x_{t-p}^{T}, e_{t-1}^{T}(\theta) \ldots e_{t-q}^{T}(\theta)\right)^{T}$, and, given 4.1), taking care that $\partial \operatorname{vec} A_{1}(\theta) / \partial \theta^{T}=\left(I_{r^{2}}, O_{r^{2}}, \ldots\right)$, $\partial \operatorname{vec} A_{2}(\theta) / \partial \theta^{T}=\left(O_{r^{2}}, I_{r^{2}}, O_{r^{2}}, \ldots\right), \ldots$, $\partial \operatorname{vec} B_{q}(\theta) / \partial \theta^{T}=\left(O_{r^{2}}, \ldots, O_{r^{2}}, I_{r^{2}}\right)$

$$
\left.B(L) \frac{\partial e_{t}(\theta)}{\partial \theta^{T}}\right|_{\theta=\theta^{0}}=-\left(X_{t-1}^{T}\left(\theta^{0}\right) \otimes I_{r}\right)
$$

We denote the derivative in the left-hand side of 4.16$)$ by $\left(\partial e_{t}\left(\theta^{0}\right)\right) /\left(\partial \theta^{T}\right)$. Let $c$ be a constant vector of the same dimension as $\theta$. Let us show that $\left(\partial e_{t}\left(\theta^{0}\right)\right) /\left(\partial \theta^{T}\right) c=0$ almost surely (a.s.) only if $c=0$. If $\left(\partial e_{t}\left(\theta^{0}\right)\right) /\left(\partial \theta^{T}\right) c=0$ a.s., then $\left(X_{t-1}^{T}\left(\theta^{0}\right) \otimes I_{r}\right) c=$ 0 a.s. but, given Assumption $\mathbf{B}_{4}$, this implies $c=0$. Given the definition of $V$ in Assumption $\mathbf{A}_{4}$, this implies that $V$, which is definite positive by construction, is invertible.

Remark 4.5. A referee pointed out that the use of ergodicity arguments can improve 
the result. The process $\left\{e_{t}(\theta): t=1,2, \ldots\right\}$ which stems from the i.i.d. sequence $\left\{\epsilon_{t}\right.$ : $t=1,2, \ldots\}$ using a time-invariant version of (3.17) is stationarity and ergodic. Then $V=\left(V_{i j}\right)_{1 \leq i, j \leq m}$ in Assumption $\mathbf{A}_{4}$ reduces to $V_{i j}=E_{\theta^{0}}\left(\left(\partial e_{t}^{T}(\theta) / \partial \theta_{i}\right) \Sigma^{-1}\left(\partial e_{t}(\theta) / \partial \theta_{j}\right)\right)$. Moreover, exploiting again the ergodicity argument, a consistent estimate of $V_{i j}$ would be $\widehat{V}_{i j}=\left.\left.\sum_{t=1}^{n}\left(\partial \widehat{e}_{t}^{T}(\theta) / \partial \theta_{i}\right)\right|_{\theta=\widehat{\theta}_{n}} \widehat{\Sigma}^{-1}\left(\partial \widehat{e}_{t}(\theta) / \partial \theta_{j}\right)\right|_{\theta=\widehat{\theta}_{n}}$, where $\widehat{e}_{t}(\theta)$ is an estimate of $e_{t}(\theta)$ using the VARMA equation (2.3) under the invertibility of the model, $\widehat{\theta}_{n}$ is the consistent estimate of $\theta^{0}$, and $\widehat{\Sigma}$ is an estimate of $\Sigma$ at $\theta=\widehat{\theta}_{n}$.

Hannan and Deistler (2012, Section 2.7) provide several more complex sufficient conditions for VARMAX models. Identifiability is not a simple concept. It is not always possible for a given parametrization. As shown by Berlinet and Francq (1998), there are processes with two different identifiable representations, one for one couple $(p, q)$ and another one for another couple. There are also VARMA processes without any identifiable representation. We will see Example 4.3 later.

Coprimeness is not easy to check - see Basilio and Kouvaritakis (1997). Hannan (1969, p. 224) mentions a sufficient (but not necessary) condition for model identifiability, that the eigenvalues of $A(z)$ all differ from the eigenvalues of $B(z)$. This assertion is given without proof and we provide an amendment below in Lemma 4.6. This is also mentioned by Dufour and Pelletier (2019), along with weaker conditions for more specific representations. In the univariate case, the eigenvalues of the two matrix polynomials are the roots of the respective $\mathrm{AR}$ and MA equations $A(z)=0$ and $B(z)=0$, and thus, a necessary and sufficient condition for existence and invertibility of the information matrix $V$ is the absence of a common root for $A(z)=0$ and $B(z)=0$. Despite the wrong conclusion in Klein et al. (2005), that property does not extend to the multivariate case.

Remark 4.6. There is indeed a mistake in the proof by Klein et al. (2005, Proposition 2.4): it is wrong to state that, if we denote a $k \times k$ matrix function $F($.$) and a k \times 1$ vector $y$, then the equality $\oint_{0}^{2 \pi} F\left(e^{i \omega}\right) y d \omega=0$ implies that $F\left(e^{i \omega}\right) y \equiv 0$, even if $F$ is a real and symmetric definite positive matrix and $y$ is in the kernel of $\oint_{0}^{2 \pi} F\left(e^{i \omega}\right) d \omega$.

First, the necessary condition is false if $r>1$. Example 4.1 shows a counterexample.

Example 4.1. Let us take the example in Klein et al. 2005, Example 1) where $\Sigma=I_{2}$ :

$$
A(z)=\left(\begin{array}{cc}
1+0.6 z & 0.2 z \\
0.4 z & 1-0.6 z
\end{array}\right), \quad B(z)=\left(\begin{array}{cc}
1+0.5 z & 0.76 z \\
0.25 z & 1-0.5 z
\end{array}\right)
$$

The two determinantal equations have the same two roots -1.50756 and 1.50756 , so if the necessary condition were true, we would have a singular information matrix. As 
mentioned in Klein et al. (2005, Section 3.1), the smallest eigenvalue of $V$ is 0.0067 instead of 0 . It is explained that inaccurate numerical calculations of integrals can be the cause and that the tensor Sylvester matrix better indicates the problem. We have mentioned that computing the Fisher information matrix for a VARMA model was first solved by Newton (1978). This was done using circular integrals of rational functions but these integrals can be evaluated by recurrence, see Söderström (1984), thus possibly without loss of accuracy. Using exact computations in Mathematica, the determinant of the unit (i.e. for $n=1$ ) information matrix for the example in 4.17) is between 808/68694 and 809/68693. Consequently, the explanation given by the authors is wrong and the necessary condition does not hold.

The determinantal equations $\operatorname{det}(A(z))=0$ and $\operatorname{det}(B(z))=0$ have respective degrees $p_{1} \leq p r$ and $q_{1} \leq q r$. If $p_{1}<p r$ and $q_{1}<q r$, we speak of missing roots. Hannan's (1969) sufficient condition on the eigenvalues holds only if the missing roots, if any, are set to infinity and the common infinity roots of the two determinantal equations are considered to be equal. This case is also not treated by Klein et al. (2005). A simpler alternative would be to consider the reciprocal polynomials $A^{*}(z)$ and $B^{*}(z)$ (see Section 3.1) and compare their eigenvalues, which are the inverses of those of $A(z)$ and $B(z)$, respectively, and should all be smaller than 1 in modulus. The missing roots of the original determinantal equations correspond to the zero eigenvalues of the reciprocal polynomials. Example 4.2 will illustrate this phenomenon.

Example 4.2. Athanasopoulos and Vahid (2008b) present a VARMA model with

$$
A(z)=\left(\begin{array}{cc}
1+A_{11} z & A_{12} z \\
0 & 1
\end{array}\right), \quad B(z)=\left(\begin{array}{cc}
1+B_{11} z & B_{12} z \\
0 & 1
\end{array}\right)
$$

They explain convincingly that the model is not identifiable without recourse to Hannan (1969)'s conditions. Here, Hannan's second condition of identifiability is violated because the matrix obtained by the juxtaposition of the matrices $A_{1}$ and $B_{1}$

$$
\left(A_{1} \vdots B_{1}\right)=\left(\begin{array}{cccc}
A_{11} & A_{12} & B_{11} & B_{12} \\
0 & 0 & 0 & 0
\end{array}\right)
$$

has rank $1<2$. But the determinants of $A(z)$ and $B(z)$ are both of degree $1<2$, hence we have common eigenvalues corresponding to the missing roots. Athanasopoulos and Vahid (2008b) mention that constraining e.g. $B_{12}$ to 0 removes the identifiability problem.

A similar example was already examined by Berlinet and Francq (1998).

Example 4.3. Berlinet and Francq (1998) indicate that the following specifications 
for any real $\alpha$ and $\beta$ are all equivalent to 4.18):

$$
A(z)=\left(\begin{array}{cc}
1+A_{11} z & \left(A_{12}+\alpha\right) z \\
0 & 1+\beta z
\end{array}\right), \quad B(z)=\left(\begin{array}{cc}
1+B_{11} z & \left(B_{12}+\alpha\right) z \\
0 & 1+\beta z
\end{array}\right) .
$$

There is no pure VAR(1) or VMA(1) identifiable representation for (4.19), as shown by Berlinet and Francq (1998). Note that (4.19) satisfies $\mathbf{B}_{4}$ ii but not $\mathbf{B}_{4}$ i because a diagonal matrix with elements 1 and $1+\beta z$ is a left common factor of $A(z)$ and $B(z)$ for $\beta \neq 0$.

Lemma 4.6 below provides the promised amended version of Hannan (1969)'s assertion on the roots of the determinantal equations.

Lemma 4.6. If Assumption $\mathbf{A}_{4}$ is not verified, then there is at least one common root for $\operatorname{det}\left(A^{*}(z)\right)=0$ and $\operatorname{det}\left(B^{*}(z)\right)=0$.

Proof of Lemma 4.6. If the lack of identifiability occurs because $\mathbf{B}_{4} \mathrm{i}$ is not satisfied, that means that there exists a non-unimodular polynomial matrix $F(z)$, i.e. with $\operatorname{det}(F(z))$ different from a constant, and matrices $A_{1}(z)$ and $B_{1}(z)$ such that $(A(z) B(z))=F(z)\left(A_{1}(z) B_{1}(z)\right)$. We have

$$
(\operatorname{det}(A(z)) \operatorname{det}(B(z)))=\operatorname{det}(F(z))\left(\operatorname{det}\left(A_{1}(z)\right) \operatorname{det}\left(B_{1}(z)\right)\right)
$$

where $\operatorname{det}(F(z))$ is a polynomial different from a constant. Hence, the equations $\operatorname{det}(A(z))=0$ and $\operatorname{det}(B(z))=0$ have at least one common root. Consequently, the matrix polynomials $A(z)$ and $B(z)$ have at least one common eigenvalue. The same is also true for the reciprocal matrix polynomials $A^{*}(z)$ and $B^{*}(z)$.

Now, if the lack of identifiability occurs because $\mathbf{B}_{4}$ ii is not satisfied, that means that the matrix $\left(A_{p} B_{q}\right)$ has rank strictly smaller than $r$. Consequently, row $r$, say, of that matrix is a linear combination of the other $r-1$ rows, and the matrices $A_{p}$ and $B_{q}$ do not have full rank. The determinants $\operatorname{det}(A(z))$ and $\operatorname{det}(B(z))$ are polynomials of degree strictly smaller than, respectively, $r p$ and $r q$. Hence the reciprocal matrix polynomials $A^{*}(z)$ and $B^{*}(z)$ have at least one zero eigenvalue, and at least one common eigenvalue.

This is simpler to check than Hannan (1969)'s $\mathbf{B}_{4}$ i and $\mathbf{B}_{4}$ ii conditions. Example 4.4 illustrates this.

Example 4.4. In Example 4.2, the determinants of the reciprocal polynomials $A^{*}(z)=$ $\left(z+A_{11}\right) z$ and $B^{*}(z)=\left(z+B_{11}\right) z$ have, respectively, the roots $\left(0,-A_{11}\right)$, and $(0$, $\left.-B_{11}\right)$, hence a common root 0 . In Example 4.3, there is a common root $-\beta$ for the determinants of the reciprocal polynomials $A^{*}(z)$ and $B^{*}(z)$. In both cases, this indicates a possible identifiability problem that is confirmed. 
Despite the errors indicated, the approach of Klein et al. (2005) is effective for comparing the eigenvalues of matrix polynomials in VARMA models. It is based on the tensor Sylvester matrix defined by Gohberg and Lerer (1976), rather than on the normal, i.e. non-tensor, Sylvester matrix, which is completely useless when $r>1$. To summarize the tensor Sylvester matrix approach, there is no common root for the two determinantal equations if, and only if, the tensor Sylvester matrix has full rank. Assuming as before that the eigenvalues of $A^{*}(z)$ and $B^{*}(z)$ are all smaller than 1 in modulus, if the tensor Sylvester matrix has maximum rank, then the model is identifiable and the Fisher information matrix $V$ exists and is strictly positive definite. As shown in the counterexample in Example 4.1, the opposite is not true. The following example illustrates the use of the tensor Sylvester matrix.

Example 4.5. In the case of Example 4.3, the tensor Sylvester matrix is the following $8 \times 8$ matrix

$$
\left(\begin{array}{cc}
-I_{4} & -B_{1} \otimes I_{2} \\
I_{4} & I_{2} \otimes A_{1}
\end{array}\right)=\left(\begin{array}{cccccccc}
-1 & 0 & 0 & 0 & -B_{11} & 0 & -B_{12}-\alpha & 0 \\
0 & -1 & 0 & 0 & 0 & -B_{11} & 0 & -B_{12}-\alpha \\
0 & 0 & -1 & 0 & 0 & 0 & -\beta & 0 \\
0 & 0 & 0 & -1 & 0 & 0 & 0 & -\beta \\
1 & 0 & 0 & 0 & A_{11} & A_{12}+\alpha & 0 & 0 \\
0 & 1 & 0 & 0 & 0 & \beta & 0 & 0 \\
0 & 0 & 1 & 0 & 0 & 0 & A_{11} & A_{12}+\alpha \\
0 & 0 & 0 & 1 & 0 & 0 & 0 & \beta
\end{array}\right) .
$$

It has clearly rank $7 \leq 8$, indicating the possible identifiability problem.

Remember, however, that in Example 4.1, the tensor Sylvester matrix would be singular although the information matrix $V$ is invertible.

Remark 4.7. There are procedures to guarantee identifiability. These include using (i) the final autoregressive representation, (ii) the echelon form, and (iii) scalar multiple components (SCM). The last two are special cases of 4.2 rather than the original VARMA equation (2.3). The echelon form is well described by Hannan and Deistler (2012), Lütkepohl (2005), and Reinsel (1997), among others. For the scalar components, see Tiao and Box (1981) or a more complete specification and estimation procedure in Athanasopoulos and Vahid (2008a). Note that Mélard et al. (2006) describe an estimation procedure that takes care of some of these particular specifications. Alternative estimation procedures based on regression are proposed by Dufour and Jouini (2014) and Dufour and Pelletier (2019), the latter for uncorrelated but non-independent innovations. 


\subsection{Proof of the main theorem}

Proof of Theorem 4.1. Of course, $\mathbf{A}_{1}$ is satisfied and since $\mathbf{B}_{1}$ coincides with $\mathbf{A}_{3}$, the other assumptions must still be checked. This is done in Lemmas 4.1, 4.3, 4.4, and 4.5 in the above subsections.

\section{Acknowledgments}

This paper begun when Christophe Ley and I were trying to complete $\operatorname{tdVAR}(1)$ theoretical examples in Alj et al. (2017). This lead me to explore an idea in Graeme McRae's blog (http://2000clicks.com/MathHelp/SeriesPolynomialGeometric.aspx). I found the crucial inspiration for Section 3 in Boubacar Maïnassara and Rabehasaina (2020). I would like to thank Yacouba Boubacar Maïnassara, Christian Francq, and Christophe Ley for their help at the early stages of this research, André Klein and Peter Spreij, for their collaboration in relation to the error in Klein et al. (2005), and Rajae Azrak and Marcella Niglio for their comments. This paper has strongly benefited from the remarks of the three referees, an associate editor, and the editor, who proposed many changes in order to improve its strength and its readability, including a complete restructuring. I would like to thank them deeply for this, and also Martin McGarry for his proofreading.

\section{References}

Alj, A., Azrak, R., Ley, C. and Mélard, G. (2017). Asymptotic properties of QML estimators for VARMA models with time-dependent coefficients, Scandinavian Journal of Statistics 44, 617-635, DOI: 10.1111/sjos.12268.

Alj, A., Azrak, R. and Mélard, G. (2020). General estimation results for tdVARMA array models, submitted.

Athanasopoulos, G., and Vahid, F. (2008a). A complete VARMA modelling methodology based on scalar components, Journal of Time Series Analysis 29(3), 533-554.

Athanasopoulos, G., and Vahid, F. (2008b). VARMA versus VAR for macroeconomic forecasting, Journal of Business and Economic Statistics 26(2), 237-252.

Azrak, R. \& Mélard, G. (2006). Asymptotic properties of quasi-likelihood estimators for ARMA models with time-dependent coefficients, Statistical Inference for Stochastic Processes 9, 279-330. 
Azrak, R. and Mélard, G. (2020). Asymptotic properties of conditional least-squares for array time series, ECARES Working Paper 2020-12. http://ecares.ulb.ac.be/index.php?.

Basilio, J. C., and Kouvaritakis, B. (1997). An algorithm for coprime matrix fraction description using Sylvester matrices, Linear Algebra and its Applications 266, 107125.

Berlinet, A. and Francq, C. (1998). On the identifiability of minimal VARMA representations, Statistical Inference for Stochastic Processes 1, 1-15.

Boubacar Maïnassara, Y. (2011). Multivariate portmanteau test for structural VARMA models with uncorrelated but non-independent error terms, Journal of Statistical Planning and Inference 141(8), 2961-2975.

Boubacar Maïnassara, Y. (2012). Selection of weak VARMA models by modified Akaike's information criteria, Journal of Time Series Analysis 33(1), 121-130.

Boubacar Maïnassara, Y. and Francq, C. (2011). Estimating structural VARMA models with uncorrelated but non-independent error terms, Journal of Multivariate Analysis 102, 496-505.

Boubacar Maïnassara, Y. and Rabehasaina, L. (2020). Estimation of weak ARMA models with regime changes, Statistical Inference for Stochastic Processes 23 1-52. DOI: $10.1007 /$ s11203-019-09202-3.

Boubacar Maïnassara, Y. and Saussereau, B. (2018). Diagnostic checking in multivariate ARMA models with dependent errors using normalized residual autocorrelations, Journal of the American Statistical Association 113(524), 1813-1827.

Brockwell, P. J. \& Davis, R. A. (1991). Time Series: Theory and Methods, Springer, New York.

Brockwell, P.J., Lindner, A. and Vollenbröker, B. (2012). Strictly stationary solutions of multivariate ARMA equations with i.i.d. noise, Annals of the Institute of Statistical Mathematics 64, 1089-1119, DOI 10.1007/s10463-012-0357-x.

Deistler, M., Dunsmuir, W. and Hannan E. J. (1978). Vector linear time series models: corrections and extensions, Advances in Applied Probability 10(2), 360-372. 
Dufour, J.-M. and Jouini, T. (2014). Asymptotic distributions for quasi-efficient estimators in echelon VARMA models, Computational Statistics and Data Analysis 73, 69-86. DOI: 10.1016/j.csda.2013.11.002.

Dufour, J.-M. and Pelletier, D. (2019). Practical methods for modelling weak VARMA processes: identification, estimation and specification with a macroeconomic application, Working Paper.

Dunsmuir, W. and Hannan, E. J. (1976). Vector linear time series models, Advances in Applied Probability 8(2), 339-364.

Francq, C. and Gautier, A. (2003). Estimation of time-varying ARMA models and applications to series subject to Markovian changes in regime. Working Paper, Université Lille 3, http://christian.francq140.free.fr/Christian-Francq/statisticseconometrics-papers/longversion.ps

Francq, C. and Gautier, A. (2004). Estimation of time-varying ARMA models with Markovian changes in regime, Statistics and Probability Letters 70, 243-251.

Francq, C. and Zakoïan, J.-M. (2001). Stationarity of multivariate Markov-switching ARMA models. Journal of Econometrics 102(2), 339-364.

Gohberg, I. and Lerer, L. (1976). Resultants of matrix polynomials, Bulletin of the American Mathematical Society 82, 565-567.

Gouriéroux, C., Monfort, A. and Renne, J.-P. (2017). Statistical inference for independent component analysis: Application to structural VAR models, Journal of Econometrics 196, 111-126.

Gouriéroux, C., Monfort, A. and Renne, J.-P. (2020). Identification and estimation in non-fundamental structural VARMA models, Review of Economic Studies 87, 1915-1953 doi:10.1093/restud/rdz028.

Hallin, M., La Vecchia, D. and Liu, H. (2020). Center-outward R-estimation for semiparametric VARMA models, Journal of the American Statistical Association, in press. doi: 10.1080/01621459.2020.1832501

Hannan E. J. (1969). The identification of vector mixed autoregressive-moving average systems, Biometrika 56(1), 223-225.

Hannan E. J. (1970). Multiple Time Series, Wiley, New York. 
Hannan E. J. (1973). The asymptotic theory of linear time-series models. Journal of Applied Probability 10(1), 130-145.

Hannan E. J. and Deistler, M. (2012). The Statistical Theory of Linear Systems, 2nd Edition, Society for Industrial and Applied Mathematics, Philadelphia.

Hannan E. J., Dunsmuir, W. T. M. and Deistler, M. (1980). Estimation of vector Armax models, Journal of Multivariate Analysis 10, 275-295.

Jeantheau, T. (1998). Strong consistency of estimators for multivariate ARCH models, Econometric Theory 14, 70-86.

Jenkins, G.M. (1979). Practical Experiences with Modelling and Forecasting Time Series, Gwilym Jenkins \& Partners Ltd., Jersey, Channel Islands.

Kascha, C. (2012). A comparison of estimation methods for vector autoregressive moving-average models. Econometric Reviews 31(3), 297-324.

Kascha, C. and Mertens, K. (2009). Business cycle analysis and VARMA models, Journal of Economic Dynamics and Control 33, 267-282.

Klein, A., Mélard, G. and Spreij, P. (2005). On the resultant property of the Fisher information matrix of a vector ARMA process, Linear Algebra and its Applications 403, 291-313.

Klimko, L. A. and Nelson, P. I. (1978). On conditional least squares estimation for stochastic processes, Annals of Statistics 6, 629-642.

Kohn, R. (1978). Asymptotic properties of time domain Gaussian estimators, Advances in Applied Probabability 10, 339-359.

Kohn, R. (1979). Asymptotic estimation and hypothesis testing results for vector linear time series models, Econometrica 47(4), 1005-1030.

Kollo, T. and von Rosen, D. (2005). Advanced Multivariate Statistics with Matrices, Springer Verlag, New York.

Ling, S and McAleer, M. (2003). Asymptotic theory for a vector ARMA-GARCH model, Econometric Theory 19, 280-310.

Lütkepohl, H. (1991). Introduction to Multiple Time Series Analysis, Springer-Verlag, New York. 
Lütkepohl, H. (2005). New Introduction to Multiple Time Series Analysis, SpringerVerlag, New York.

Lütkepohl, H. (2006). Forecasting with VARMA Models, Chapter 6 in G. Elliott, C.W.J. Granger, A. Timmermann (Eds), Handbook of Economic Forecasting, Volume 1, pp. 287-325.

Mélard, G., Roy, R. and Saidi, A. (2006). Exact maximum likelihood estimation of structured or unit root multivariate time series models, Computational Statistics and Data Analysis 50(11), 2958-2986. DOI information: 10.1016/j.csda.2005.06.009.

Newton, H. J. (1978). The information matrices of the parameters of multiple mixed time series, Journal of Multivariate Analysis 8 317-323.

Pollock, D. S. G. (1999). A Handbook of Time-Series Analysis, Signal Processing and Dynamics, Academic Press, London.

Poskitt, D. S. (2005). A note on the specification and estimation of ARMAX systems, Journal of Time Series Analysis 26, 157-183.

Pötcher, B. M. (1987). Convergence results for maximum likelihood type estimators in multivariable ARMA models, Journal of Multivariate Analysis 21, 29-52.

Reinsel, G. C. (1997). Elements of Multivariate Time Series Analysis, 2nd edition, Springer-Verlag, New-York.

Rissanen, J. and Caines, P. E. (1979). The strong consistency of maximum likelihood estimators for ARMA processes, Annals of Statistics 7(2), 297-315.

Roy, A., McElroy, T. S. and Linton, P. (2019). Constrained estimation of causal invertible VARMA, Statistica Sinica 29, 455-478.

Ruiz, S. M. (1996). An algebraic identity leading to Wilson's theorem, The Mathematical Gazette 80(489), 579-582.

Stelzer, R. (2009). On Markov-switching ARMA processes - stationarity, existence of moments, and geometric ergodicity, Econometric Theory 25(1), 43-62.

Söderström, T. (1984). Description of a program for integrating rational functions around the unit circle. Technical Report 8467R, Department of Technology. Uppsala University, 1984. 
Tiao, G. C. and Box, G. E. P. (1981). Modeling multiple timeseries with applications, Journal of the American Statistical Association 76, 802-816.

Tsay, R. S. (2014). Multivariate Time Series Analysis: with $R$ and Financial Applications, Wiley, Hoboken (New Jersey).

Wei, W. W. S. (2006). Time Series Analysis: Univariate and Multivariate Methods, 2nd edition, Pearson, Boston.

Wegge, L. L. (2012). ARMAX(p,r,q) parameter identifiability without coprimeness, Working Paper, No. 12-17, University of California, Department of Economics, Davis, CA.

Whittle, P. (1953a). Estimation and information in stationary time series, Arkiv för Mathematik 2(23), 423-434.

Whittle, P. (1953b). The analysis of multiple stationary time series, Journal of the Royal Statistical Society Series B 15, 125-139.

Wilms, I., Basu, S, Bien, J. and Matteson, D. S. (2019). Sparse identification and estimation of large-scale vector autoregressive moving averages, Working paper.

Yao, Q. and Brockwell, P. J. (2006). Gaussian maximum likelihood estimation for ARMA models. I. Time series, Journal of Time Series Analysis 27, 857-875.

Yozgatligil, C. and Wei, W. W. S. (2009). Representation of multiplicative seasonal vector autoregressive moving average models, The American Statistician 63(4), 328-334. 


\section{A. Supplementary Material. From tdARMA models to ARMA models}

\section{A.1. Introduction}

This appendix is intended as a light version of the main paper specialized to univariate models, hence with $r=1$. Roman-numbered references are from the main text.

This text is heavily based on Azrak and Mélard (2006), but also refers to a theorem of Azrak and Mélard (2020). The proofs are also more detailed. We have also corrected a certain number of mistakes, including those mentioned in Remark 1 of Alj et al. (2017). We also point out that we need moments of order 4 for the errors, not moments of order 8, as required in Azrak and Mélard (2006) and Alj et al. (2017).

\section{A.2. Preliminaries for tdARMA models}

Let us consider the limited Taylor expansion of $Q_{n}(\theta)=\frac{1}{2} \sum_{t=1}^{n} \alpha_{t}(\theta)$ around $\theta^{0}$, where $^{T}$ denotes transposition

$$
Q_{n}(\theta)=Q_{n}\left(\theta^{0}\right)+\left.\left(\theta-\theta^{0}\right)^{T} \frac{\partial Q_{n}(\theta)}{\partial \theta}\right|_{\theta=\theta^{0}}+\left.\frac{1}{2}\left(\theta-\theta^{0}\right)^{T} \frac{\partial^{2} Q_{n}(\theta)}{\partial \theta \partial \theta^{T}}\right|_{\theta=\theta^{\prime}}\left(\theta-\theta^{0}\right),
$$

where $\theta^{\prime}$ is an intermediate point. We will use Klimko and Nelson (1978) theorems, denoted KN below, in a single theorem. Let $\|$.$\| denote the norm of a vector, and \stackrel{L}{\rightarrow}$ indicate convergence in law.

\section{Theorem}

Assume that

$\mathrm{i} \lim _{n \rightarrow \infty \Delta \downarrow 0} \frac{1}{n \Delta}\left(\left|\frac{\partial^{2} Q_{n}(\theta)}{\partial \theta \partial \theta^{T}}\right|_{\theta=\theta^{\prime}}-\left.\frac{\partial^{2} Q_{n}(\theta)}{\partial \theta \partial \theta^{T}}\right|_{\theta=\theta^{0}} \mid\right)<\infty$, where $\theta^{\prime}$ is such that $\left\|\theta^{\prime}-\theta^{0}\right\|<$ $\Delta$,

ii $\lim _{\substack{n \rightarrow \infty \\ \text { matrix }}} \frac{1}{2 n} E_{\theta^{0}}\left(\frac{\partial^{2} Q_{n}(\theta)}{\partial \theta \partial \theta^{T}} / F_{t-1}\right)={ }_{\text {def }} V$, a.s. where $V$ is a strictly positive definite iii $\left.\lim _{n \rightarrow \infty} \frac{1}{n} \frac{\partial Q_{n}(\theta)}{\partial \theta^{T}}\right|_{\theta=\theta^{0}}=0$, a.s.,

iv $\left.\frac{1}{\sqrt{n}} \frac{\partial Q_{n}(\theta)}{\partial \theta^{T}}\right|_{\theta=\theta^{0}} \stackrel{L}{\longrightarrow} N(0, W)$ when $n \rightarrow \infty$, where $W$ is a positive definite matrix defined by

$$
W=\lim _{n \rightarrow \infty} \frac{1}{4 n} \sum_{t=1}^{n} E_{\theta^{0}}\left(\frac{\partial \alpha_{t}(\theta)}{\partial \theta} \frac{\partial \alpha_{t}(\theta)}{\partial \theta^{T}}\right)
$$

Let $\hat{\theta}_{n}=\underset{\theta \in B}{\operatorname{argmin}} Q_{n}(\theta)$. Then, when $n \rightarrow \infty, \hat{\theta}_{n} \rightarrow \theta^{0}$, a.s., and $n^{1 / 2}\left(\hat{\theta}_{n}-\theta^{0}\right) \stackrel{L}{\longrightarrow}$ $N\left(0, V^{-1} W V^{-1}\right)$. 
Note that KNs assumption i in their Theorem 2.1 is simplified here.

Here is Theorem 1 of Azrak and Mélard (2006) with the exception that their Assumption $\mathbf{C}_{1}$ is lighter here and is taken from Azrak and Mélard (2020). Instead of $\alpha_{t}(\theta)=e_{t}^{2}(\theta) / \sigma^{2}$, we use

$$
\alpha_{t}(\theta)=e_{t}^{2}(\theta),
$$

so that $V$ here corresponds to $V / \sigma^{2}$ in the main paper.

Theorem A.1. Let the stochastic process $\left\{x_{t} ; t=1,2, \ldots\right\}$ be such that $E_{\theta}\left|x_{t}\right|^{2}<\infty$ for all $\theta$ and such that $\hat{x}_{t / t-1}(\theta)$ is almost surely (a.s.) twice continuously differentiable in an open subset $\Theta$ which contains the true value $\theta^{0}$ of vector $\theta$. Suppose that $\epsilon_{t}$ is uncorrelated with $\hat{x}_{t / t-1}\left(\theta^{0}\right)$, and

$\mathbf{C}_{1}$ : there exists $C_{1}>0$ and $\delta>0$ such that for all $t \geq 1$ :

$$
E_{\theta^{0}}\left|\frac{\partial \alpha_{t}(\theta)}{\partial \theta_{j}}\right|^{2+\delta} \leq C_{1} \quad \text { a.s. }, \quad j=1, \ldots, m
$$

$\mathrm{C}_{2}$ : there exists $C_{2}>0$ such that for all $t \geq 1$ :

$$
E_{\theta^{0}}\left|\frac{\partial^{2} \alpha_{t}(\theta)}{\partial \theta_{i} \partial \theta_{j}}-E_{\theta}\left(\frac{\partial^{2} \alpha_{t}(\theta)}{\partial \theta_{i} \partial \theta_{j}} / F_{t-1}\right)\right|^{2} \leq C_{2} \quad \text { a.s. }, \quad i, j=1, \ldots, m
$$

$\mathbf{C}_{3}: \quad \lim _{n \rightarrow \infty} \frac{1}{2 n} \sum_{t=1}^{n} E_{\theta^{0}}\left(\frac{\partial^{2} \alpha_{t}(\theta)}{\partial \theta_{i} \partial \theta_{j}} / F_{t-1}\right)=V_{i j} \quad$ a.s., $\quad i, j=1, \ldots, m$,

where the matrix $V$ is a strictly definite positive matrix of constants;

$\mathbf{C}_{4}: \quad \lim _{n \rightarrow \infty} \sup _{\Delta \downarrow 0} \frac{1}{n \Delta}\left|\sum_{t=1}^{n}\left(\left.\frac{\partial^{2} \alpha_{t}(\theta)}{\partial \theta_{i} \partial \theta_{j}}\right|_{\theta=\theta^{*}}-\left.\frac{\partial^{2} \alpha_{t}(\theta)}{\partial \theta_{i} \partial \theta_{j}}\right|_{\theta=\theta^{0}}\right)\right|<\infty, \quad$ a.s., $\quad i, j=1, \ldots, m$,

where $\theta^{*}$ is a point of the straight line joining $\theta^{0}$ to every $\theta$, such that, $\left\|\theta-\theta^{0}\right\|<\Delta$. Then, $\hat{\theta}_{n} \rightarrow \theta^{0}$ a.s. and such that for every $\epsilon>0$, there exists an event $E$ in $\left(\Omega, F, P_{\theta^{0}}\right)$ with $P_{\theta^{0}}(E)>1-\epsilon$ and $n_{0}$ such that for $n>n_{0}$, in $E, \partial Q_{n}\left(\hat{\theta}_{n}\right) / \partial \theta_{i}=0, i=1, \ldots, m$, and $Q_{n}(\theta)$ reaches a relative maximum at the point $\hat{\theta_{n}}$.

If, furthermore, we make the following assumptions:

$$
\mathbf{C}_{5}: \frac{1}{n} \sum_{t=1}^{n} E_{\theta^{0}}\left(\frac{\partial \alpha_{t}(\theta)}{\partial \theta} \frac{\partial \alpha_{t}(\theta)}{\partial \theta^{T}} / F_{t-1}\right)-\frac{1}{n} \sum_{t=1}^{n} E_{\theta^{0}}\left(\frac{\partial \alpha_{t}(\theta)}{\partial \theta} \frac{\partial \alpha_{t}(\theta)}{\partial \theta^{T}}\right) \rightarrow 0 \quad \text { a.s. },
$$

as $n \rightarrow \infty$; 
$\mathbf{C}_{6}$ : the following matrix $W$ exists

$$
W=\lim _{n \rightarrow \infty} \frac{1}{4 n} \sum_{t=1}^{n} E_{\theta^{0}}\left(\frac{\partial \alpha_{t}(\theta)}{\partial \theta} \frac{\partial \alpha_{t}(\theta)}{\partial \theta^{T}}\right)
$$

then

$$
n^{1 / 2}\left(\hat{\theta}_{n}-\theta^{0}\right) \stackrel{L}{\rightarrow} N\left(0, V^{-1} W V^{-1}\right), \text { when } n \rightarrow \infty .
$$

Proof of Theorem 1

To check KN's assumption iii for $Q_{n}(\theta)$, we first note that $\left\{\partial \alpha_{t}(\theta) / \partial \theta_{i}, F_{t}\right\}$ is a martingale difference sequence since $\partial \alpha_{t}(\theta) / \partial \theta_{i}$ belongs to $F_{t}$ and is integrable, and

$$
\frac{\partial \alpha_{t}(\theta)}{\partial \theta_{i}}=2 e_{t}(\theta) \frac{\partial e_{t}(\theta)}{\partial \theta_{i}}
$$

To apply a strong law of large numbers for martingales (Stout, 1974, p. 154, Theorem 3.3.8), the following condition is checked

$$
\sum_{t=1}^{\infty} \frac{E_{\theta^{0}}\left|\partial \alpha_{t}(\theta) / \partial \theta_{i}\right|^{p}}{t^{1+p / 2}}<\infty
$$

for $p \geq 2$. We use $\mathbf{C}_{1}$ and take $p=2+\delta$ so that

$$
\sum_{t=1}^{\infty} \frac{E_{\theta^{0}}\left|\partial \alpha_{t}(\theta) / \partial \theta_{i}\right|^{2+\delta}}{t^{2+\delta / 2}} \leq C_{1} \sum_{t=1}^{\infty} \frac{1}{t^{2+\delta / 2}}<\infty .
$$

Hence it is true that

$$
\left.\frac{1}{n} \frac{\partial Q_{n}(\theta)}{\partial \theta_{i}}\right|_{\theta=\theta^{0}} \rightarrow 0 \quad \text { a.s., } \quad i=1, \ldots, m .
$$

For KN's assumption ii, we evaluate the second derivative of $\alpha_{t}(\theta)$ defined by (A.1):

$$
\frac{\partial^{2} \alpha_{t}(\theta)}{\partial \theta_{i} \partial \theta_{j}}=2 \frac{\partial e_{t}(\theta)}{\partial \theta_{i}} \frac{\partial e_{t}(\theta)}{\partial \theta_{j}}+2 e_{t}(\theta) \frac{\partial^{2} e_{t}(\theta)}{\partial \theta_{i} \partial \theta_{j}},
$$

$i, j=1, \ldots, m$. Hence

$$
E_{\theta}\left(\frac{\partial^{2} \alpha_{t}(\theta)}{\partial \theta_{i} \partial \theta_{j}} / F_{t-1}\right)=2 \frac{\partial e_{t}(\theta)}{\partial \theta_{i}} \frac{\partial e_{t}(\theta)}{\partial \theta_{j}}
$$

and it is clear that

$$
\frac{\partial^{2} \alpha_{t}(\theta)}{\partial \theta_{i} \partial \theta_{j}}-E_{\theta}\left(\frac{\partial^{2} \alpha_{t}(\theta)}{\partial \theta_{i} \partial \theta_{j}} / F_{t-1}\right)=2 e_{t}(\theta) \frac{\partial^{2} e_{t}(\theta)}{\partial \theta_{i} \partial \theta_{j}}
$$

defines a martingale difference sequence. Using $\mathbf{C}_{2}$, we use the same law of large 
numbers for martingale sequences but with $p=2$,

$$
\sum_{t=1}^{\infty} \frac{1}{t^{2}} E_{\theta^{0}}\left|\frac{\partial^{2} \alpha_{t}(\theta)}{\partial \theta_{i} \partial \theta_{j}}-E_{\theta^{0}}\left(\frac{\partial^{2} \alpha_{t}(\theta)}{\partial \theta_{i} \partial \theta_{j}} / F_{t-1}\right)\right|^{2}<\sum_{t=1}^{\infty} \frac{C_{2}}{t^{2}}<\infty
$$

hence

$$
\left.\frac{1}{n} \sum_{t=1}^{n} \frac{\partial^{2} \alpha_{t}(\theta)}{\partial \theta_{i} \partial \theta_{j}}\right|_{\theta=\theta^{0}}-\frac{1}{n} \sum_{t=1}^{n} E_{\theta^{0}}\left(\frac{\partial^{2} \alpha_{t}(\theta)}{\partial \theta_{i} \partial \theta_{j}} / F_{t-1}\right) \rightarrow 0 \quad \text { a.s. }
$$

$\mathbf{C}_{3}$ implies that

$$
\lim _{n \rightarrow \infty} \frac{1}{2 n} \sum_{t=1}^{n} E_{\theta^{0}}\left(\frac{\partial^{2} \alpha_{t}(\theta)}{\partial \theta_{i} \partial \theta_{j}} / F_{t-1}\right)=V_{i j}
$$

with $V$ a strictly definite positive matrix, checking thus KN's assumption ii.

Consistency is proved because $\mathbf{C}_{4}$ is identical to KN's assumption i. We use now a central limit theorem to verify KN's assumption iv. We will prove that

$$
\left.n^{1 / 2} \frac{\partial Q_{n}(\theta)}{\partial \theta}\right|_{\theta=\theta^{0}} \stackrel{L}{\rightarrow} N(0, W)
$$

if $n \rightarrow \infty$, using the Cramér-Wold device. We denote by $\lambda$ a vector of dimension $m \times 1$ and $\xi_{t}=\lambda^{T} \partial \alpha_{t}(\theta) /\left.\partial \theta\right|_{\theta=\theta^{0}}$. for all $\lambda$. To show the asymptotic normality of $\sum_{t=1}^{n} \xi_{t}$ for all $\lambda$, we check the assumptions of Basawa and Prakasa Rao s theorem (Basawa and Prakasa Rao, 1980, p. 388) for $\xi_{t}$. Knowing that $E_{\theta^{0}}\left(\xi_{t} / F_{t-1}\right)=0$, let us now check that there exists $\Delta_{1}>0$ and $M(\lambda)$, such that $E\left|\xi_{t}\right|^{2+\Delta_{1}} \leq M(\lambda)<\infty$, $\forall t \geq 1$. Let $\|\cdot\|_{\infty}$ denote the maximum norm such that $\|\lambda\|_{\infty}=\max _{i=1, \ldots, m}\left|\lambda_{i}\right|$. For $\Delta_{1}=\delta>0$ in $\mathbf{C}_{1}$, we have

$$
\begin{aligned}
\left|\xi_{t}\right| & \leq\|\lambda\|_{\infty} \sum_{i=1}^{m}\left|\frac{\partial \alpha_{t}(\theta)}{\partial \theta_{i}}\right| \\
& \leq\|\lambda\|_{\infty}\left(\sum_{i=1}^{m}\left|\frac{\partial \alpha_{t}(\theta)}{\partial \theta_{i}}\right|^{2+\delta}\right)^{\frac{1}{2+\delta}} m^{\frac{1+\delta}{2+\delta}}
\end{aligned}
$$

using Hölder inequality for sums with Hölder conjugates $p=2+\delta$ and $q=(2+$ $\delta) /(1+\delta)$. Hence

$$
\begin{aligned}
E_{\theta^{0}}\left|\xi_{t}\right|^{2+\delta} & \leq m^{1+\delta}\|\lambda\|_{\infty}^{2+\delta} \sum_{i=1}^{m} E_{\theta^{0}}\left|\frac{\partial \alpha_{t}(\theta)}{\partial \theta_{i}}\right|^{2+\delta} \\
& \leq m^{2+\delta}\|\lambda\|_{\infty}^{2+\delta} C_{1}<\infty
\end{aligned}
$$

so that the first condition is verified. Now we show that

$$
\lim _{n \rightarrow \infty} \frac{1}{n} \sum_{t=1}^{n} E_{\theta^{0}}\left(\xi_{t}^{2} / F_{t-1}\right)=\gamma^{2}(\lambda)
$$


where $\gamma^{2}(\lambda) \geq 0$. Because of $\mathbf{C}_{5}$, A.10 equals

$$
\begin{gathered}
\lim _{n \rightarrow \infty} \frac{1}{n} \sum_{t=1}^{n} E_{\theta^{0}}\left(\lambda^{T} \frac{\partial \alpha_{t}(\theta)}{\partial \theta} \frac{\partial \alpha_{t}(\theta)}{\partial \theta^{T}} \lambda / F_{t-1}\right) \\
=\lim _{n \rightarrow \infty} \frac{1}{n} \sum_{t=1}^{n} E_{\theta^{0}}\left(\lambda^{T} \frac{\partial \alpha_{t}(\theta)}{\partial \theta} \frac{\partial \alpha_{t}(\theta)}{\partial \theta^{T}} \lambda\right)=4 \lambda^{T} W \lambda \geq 0 .
\end{gathered}
$$

This is our expression for $\gamma^{2}(\lambda)$. We can apply the Basawa and Prakasa Rao (1980) theorem to obtain

$$
n^{1 / 2} \sum_{t=1}^{n} \xi_{t} \stackrel{L}{\rightarrow} N\left(0, \gamma^{2}(\lambda)\right) \quad \text { or }\left.\quad n^{1 / 2} \lambda^{T} \frac{\partial Q_{n}(\theta)}{\partial \theta}\right|_{\theta=\theta^{0}} \stackrel{L}{\rightarrow} N\left(0, \lambda^{T} W \lambda\right),
$$

when $n \rightarrow \infty$. Because this is valid for all values of $\lambda$, we have proved A.9.

\section{A.3. The asymptotic theory for homoscedastic tdARMA models}

In this section, which expands and provides more detail in relation to Section 3.3 of the main paper, Theorem A.1 is applied to homoscedastic tdARMA models, i.e. (2.2) with $r=1$. Theorem A.2 is the scalar version of Theorem 3.1 in the main paper. The notations at the end of its Section 3.1 and at the beginning of Section 3.2 are not repeated, especially those in $(3.9),(3.19-3.21)$, where the $\psi_{t k}, \psi_{t i k}, \psi_{t i j k}$, and $\psi_{t i j l k}$ are defined. We use the following lemma from Azrak and Mélard (2006, Lemma 1).

Lemma A.1. Let $\left(w_{t}, t \in Z\right)$ be a process with second-order moments which are uniformly bounded with respect to $t$, and let $\nu>0$ be such that

$$
E\left(\frac{1}{n} \sum_{t=1}^{n} w_{t}\right)^{2}=O\left(\frac{1}{n^{\nu}}\right)
$$

Then, $n^{-1} \sum_{t=1}^{n} w_{t}$ converges almost surely to zero when $n$ tends to infinity.

Theorem A.2. Consider an autoregressive-moving average process defined by (2.2) for $r=1$. and suppose that for all $t=1, \ldots, n$ :

$\mathbf{A}_{1}: A_{i, t}(\theta), i=1, \ldots, p$, and $B_{j, t}(\theta), j=1, \ldots, q$ are three times continuously differentiable with respect to $\theta$, in the open set $B$ containing the true value $\theta^{0}$ of $\theta$, and

$\mathbf{A}_{2}$ : there exist positive constants $N_{\ell}, \ell=1, \ldots, 7$, and $0<\Phi<1$, such that for $\nu=1, \ldots, t-1$ and $i, j, l=1, \ldots, m$ :

$$
\begin{array}{ll}
\sum_{k=\nu}^{t-1} \psi_{t i k}^{2}<N_{1} \Phi^{\nu-1}, & \sum_{k=\nu}^{t-1} \psi_{t i k}^{4}<N_{2} \Phi^{\nu-1} \\
\sum_{k=\nu}^{t-1} \psi_{t i j k}^{2}<N_{3} \Phi^{\nu-1}, & \sum_{k=\nu}^{t-1} \psi_{t i j k}^{4}<N_{4} \Phi^{\nu-1}
\end{array}
$$




$$
\sum_{k=1}^{t-1} \psi_{t i j l k}^{2}<N_{5}, \quad \sum_{k=1}^{t-1} \psi_{t k}^{2}<N_{6}, \quad \sum_{k=1}^{t-1} \psi_{t k}^{4}<N_{7}
$$

$\mathbf{A}_{3}$ : there exists $E\left(\epsilon_{t}^{4}\right)=K_{4}<\infty$ and hence $\kappa=K_{4}-3$;

$\mathbf{A}_{4}: \quad \lim _{n \rightarrow \infty} \frac{1}{n} \sum_{t=1}^{n}\left[E_{\theta^{0}}\left(\frac{\partial e_{t}(\theta)}{\partial \theta_{i}} \frac{\partial e_{t}(\theta)}{\partial \theta_{j}}\right)\right]=V_{i j}, \quad i, j=1, \ldots, m$,

where the matrix $V$ is a strictly definite positive matrix;

$\mathbf{A}_{5}$ : for $i, j=1, \ldots, m$, we have

$$
\frac{1}{n^{2}} \sum_{d=1}^{n-1} \sum_{t=1}^{n-d} \sum_{k=1}^{t-d} \psi_{t i k} \psi_{t+d, j, k+d}=O\left(\frac{1}{n}\right)
$$

$\mathbf{A}_{6}:$ for $i, j=1, \ldots, m$, we have

$$
\frac{1}{n^{2}} \sum_{d=1}^{n-1} \sum_{t=1}^{n-d} \sum_{k=1}^{t-1-d} \psi_{t i k} \psi_{t j k} \psi_{t+d, i, k+d} \psi_{t+d, j, k+d}=O\left(\frac{1}{n}\right)
$$

Then, when $n \rightarrow \infty$,

- there exists an estimator $\hat{\theta}_{n}$ such that $\hat{\theta}_{n} \rightarrow \theta^{0}$ almost surely and in the sense of Theorem A.1

- $n^{1 / 2}\left(\hat{\theta}_{n}-\theta^{0}\right) \stackrel{L}{\rightarrow} N\left(0, V^{-1}\right)$.

Proof of Theorem A.2

We check the assumptions $\mathbf{C}_{1}-\mathbf{C}_{5}$ of Theorem A.1. Note that $\epsilon_{t}$ is independent of $\left.\left(\partial e_{t}(\theta) / \partial \beta_{i}\right)\right|_{\theta=\theta^{0}}, i=1, \ldots, m$.

Proof of $\mathbf{C}_{1}$. Let us show there is a positive constant $C_{1}$ such that $(\mathrm{A} .2)$ holds. From A.6.

$$
\left|\frac{\partial \alpha_{t}(\theta)}{\partial \theta_{i}}\right|^{2+\delta}=2^{2+\delta}\left|e_{t}(\theta)\right|^{2+\delta}\left|\frac{\partial e_{t}(\theta)}{\partial \theta_{i}}\right|^{2+\delta} .
$$

The expectation at $\theta=\theta^{0}$ is bounded by $2^{2+\delta}$ times

$$
E_{\theta^{0}}\left(\left|e_{t}(\theta)\right|^{2+\delta}\left|\frac{\partial e_{t}(\theta)}{\partial \theta_{i}}\right|^{2+\delta}\right)=E_{\theta^{0}}\left(\left|e_{t}(\theta)\right|^{2+\delta}\right) E_{\theta^{0}}\left(\left|\frac{\partial e_{t}(\theta)}{\partial \theta_{i}}\right|^{2+\delta}\right)
$$

We use Lyapunov inequality, that for $0<r \leq s$ and any random variable $X$, $\left(E|X|^{r}\right)^{1 / r} \leq\left(E|X|^{s}\right)^{1 / s}$, provided the latter expectation exists, to show that the expression A.11 is bounded. The first factor is bounded by $\left(E\left|\epsilon_{t}\right|^{2+\delta}\right) \leq\left(E \epsilon_{t}^{4}\right)^{(2+\delta) / 4}$ which exists thanks to $\mathbf{A}_{4}$. Similarly for the second factor of A.11 which is bounded by

$$
\left(E_{\theta^{0}}\left|\frac{\partial e_{t}(\theta)}{\partial \theta_{i}}\right|^{4}\right)^{(2+\delta) / 4}
$$


The expectation is a special case for $j=i$ of

$$
E_{\theta^{0}}\left\{\left(\frac{\partial e_{t}(\theta)^{2}}{\partial \theta_{i}}\right)\left(\frac{\partial e_{t}(\theta)}{\partial \theta_{j}}\right)^{2}\right\}
$$

whose expression will be used later, and this equals, given (3.19),

$$
\sum_{k_{1}} \sum_{k_{2}} \sum_{k_{3}} \sum_{k_{4}} \psi_{t i k_{1}} \psi_{t i k_{2}} \psi_{t j k_{3}} \psi_{t j k_{4}} E\left(\epsilon_{t-k_{1}} \epsilon_{t-k_{2}} \epsilon_{t-k_{3}} \epsilon_{t-k_{4}}\right),
$$

where the sums over $k_{u}, u=1, \ldots, 4$ are from 1 to $t-1$. Because of the independence of the $\epsilon_{t}$ 's, and $E\left(\epsilon_{t}\right)=0$, this is equal to

$$
\begin{gathered}
\sum_{k=1}^{t-1} \psi_{t i k}^{2} \psi_{t j k}^{2} E\left(\epsilon_{t-k}^{4}\right)+\sigma^{4}\left\{\sum_{\substack{k_{1} \\
k_{1} \neq k_{2}}} \sum_{k_{2}} \psi_{t i k_{1}} \psi_{t i k_{2}} \psi_{t j k_{1}} \psi_{t j k_{2}}\right. \\
\left.\quad+\sum_{\substack{k_{1} \\
k_{1} \neq k_{3}}} \sum_{k_{3}} \psi_{t i k_{1}}^{2} \psi_{t j k_{3}}^{2}+\sum_{\substack{k_{1} \\
k_{1} \neq k_{2}}} \sum_{k_{2}} \psi_{t i k_{1}} \psi_{t i k_{2}} \psi_{t j k_{2}} \psi_{t j k_{1}}\right\} .
\end{gathered}
$$

Hence, by completing the double sums for $k_{1}=k_{2}$, we have

$$
\begin{aligned}
E_{\theta^{0}}\left\{\left(\frac{\partial e_{t}(\theta)}{\partial \theta_{i}}\right)^{2}\left(\frac{\partial e_{t}(\theta)}{\partial \theta_{j}}\right)^{2}\right\}= & \sum_{k=1}^{t-1} \psi_{t i k}^{2} \psi_{t j k}^{2} E\left(\epsilon_{t-k}^{4}\right)+\sigma^{4}\left\{\sum_{k_{1}} \sum_{k_{3}} \psi_{t i k_{1}}^{2} \psi_{t j k_{3}}^{2}\right. \\
& \left.+\sum_{k_{1}} \sum_{k_{2}} \psi_{t i k_{1}} \psi_{t i k_{2}} \psi_{t j k_{1}} \psi_{t j k_{2}}+\sum_{k_{1}} \sum_{k_{2}} \psi_{t i k_{1}} \psi_{t i k_{2}} \psi_{t j k_{2}} \psi_{t j k_{1}}\right\} \\
& -\sigma^{4}\left\{\sum_{k=1}^{t-1} \psi_{t i k}^{2} \psi_{t j k}^{2}+\sum_{k=1}^{t-1} \psi_{t i k} \psi_{t i k} \psi_{t j k} \psi_{t j k}+\sum_{k=1}^{t-1} \psi_{t i k} \psi_{t i k} \psi_{t j k} \psi_{t j k}\right\} \\
= & \sum_{k=1}^{t-1} \psi_{t i k}^{2} \psi_{t j k}^{2} E\left(\epsilon_{t-k}^{4}\right)+\sigma^{4}\left\{\left(\sum_{k=1}^{t-1} \psi_{t i k}^{2}\right)\left(\sum_{k=1}^{t-1} \psi_{t j k}^{2}\right)\right. \\
+ & \left.2\left(\sum_{k=1}^{t-1} \psi_{t i k} \psi_{t i k}\right)^{2}\right\}-3 \sigma^{4}\left\{\sum_{k=1}^{t-1} \psi_{t i k}^{2} \psi_{t j k}^{2}\right\} \\
= & \kappa \sum_{k=1}^{t-1} \psi_{t i k}^{2} \psi_{t j k}^{2}+\sigma^{4}\left\{\left(\sum_{k=1}^{t-1} \psi_{t i k}^{2}\right)\left(\sum_{k=1}^{t-1} \psi_{t j k}^{2}\right)+2\left(\sum_{k=1}^{t-1} \psi_{t i k} \psi_{t i k}\right)^{2}\right\} \\
\leq & \kappa N_{2}+3 \sigma^{4} N_{1}^{2} .
\end{aligned}
$$

Hence (A.13) is bounded from above and the upper bound A.2 is checked. 
Proof of $\mathbf{C}_{2}$. For the conditional expectation of $(A .8)$ at $\theta=\theta^{0}$, we have

$$
E_{\theta^{0}}\left|\frac{\partial^{2} \alpha_{t}(\theta)}{\partial \theta_{i} \partial \theta_{j}}-E_{\theta^{0}}\left(\frac{\partial^{2} \alpha_{t}(\theta)}{\partial \theta_{i} \partial \theta_{j}} / F_{t-1}\right)\right|^{2}=E_{\theta^{0}}\left|2 e_{t}(\theta) \frac{\partial^{2} e_{t}(\theta)}{\partial \theta_{i} \partial \theta_{j}}\right|^{2}, \quad i, j=1, \ldots, m .
$$

This is equal to

$$
4 E_{\theta^{0}}\left(e_{t}^{2}(\theta)\right) E_{\theta^{0}}\left|\frac{\partial^{2} e_{t}(\theta)}{\partial \theta_{i} \partial \theta_{j}}\right|^{2}=4 \sigma^{2} \sum_{k=1}^{t-1} \psi_{t i j k}^{2}
$$

using this time (3.20) for $\theta=\theta^{0}$, and this is smaller than $4 \sigma^{2} N_{3}$, which is the value of the constant $C_{2}$ in $\mathrm{A} .2$.

Proof of $\mathrm{C}_{3}$. We start from

$$
\frac{1}{2 n} \sum_{t=1}^{n} E_{\theta^{0}}\left(\frac{\partial^{2} \alpha_{t}(\theta)}{\partial \theta_{i} \partial \theta_{j}} / F_{t-1}\right)=\left.\frac{1}{n} \sum_{t=1}^{n} \frac{\partial e_{t}(\theta)}{\partial \theta_{i}} \frac{\partial e_{t}(\theta)}{\partial \theta_{j}}\right|_{\theta=\theta^{0}} .
$$

For $i, j=1, \ldots, m$, we consider the process $Z_{t, i j}$ defined by

$$
Z_{t, i j}=\left.\left.\frac{\partial e_{t}(\theta)}{\partial \theta_{i}}\right|_{\theta=\theta^{0}} \frac{\partial e_{t}(\theta)}{\partial \theta_{j}}\right|_{\theta=\theta^{0}}-E_{\theta^{0}}\left(\frac{\partial e_{t}(\theta)}{\partial \theta_{i}} \frac{\partial e_{t}(\theta)}{\partial \theta_{j}}\right)
$$

and show that the conditions of Lemma A.1 are satisfied. First

$$
E\left(Z_{t, i j}^{2}\right) \leq E_{\theta^{0}}\left(\frac{\partial e_{t}(\theta)}{\partial \theta_{i}} \frac{\partial e_{t}(\theta)}{\partial \theta_{j}}\right)^{2}
$$

This expression is the same as in $(\mathrm{A} .12)$, for which we found an upper bound. Second, let us show that

$$
E_{\theta^{0}}\left(\frac{1}{n} \sum_{t=1}^{n} Z_{t, i j}\right)^{2}=\operatorname{var}_{\theta^{0}}\left(\frac{1}{n} \sum_{t=1}^{n} \frac{\partial e_{t}(\theta)}{\partial \theta_{i}} \frac{\partial e_{t}(\theta)}{\partial \theta_{j}}\right)=O\left(\frac{1}{n}\right) .
$$

This is equal to

$$
\frac{1}{n^{2}} \sum_{d=1}^{n-1} \sum_{t=1}^{n-d} \operatorname{cov}_{\theta^{0}}\left(\frac{\partial e_{t}(\theta)}{\partial \theta_{i}} \frac{\partial e_{t}(\theta)}{\partial \theta_{j}}, \frac{\partial e_{t+d}(\theta)}{\partial \theta_{i}} \frac{\partial e_{t+d}(\theta)}{\partial \theta_{j}}\right)
$$

and the covariance is written as

$$
\begin{aligned}
\sum_{k_{1}} \sum_{k_{2}} \sum_{k_{3}} \sum_{k_{4}} \psi_{t i k_{1}} \psi_{t j k_{2}} \psi_{t+d, i, k_{3}} \psi_{t+d, j, k_{4}} & \left\{E\left(\epsilon_{t-k_{1}} \epsilon_{t-k_{2}} \epsilon_{t+d-k_{3}} \epsilon_{t+d-k_{4}}\right)-E\left(\epsilon_{t-k_{1}} \epsilon_{t-k_{2}}\right) E\left(\epsilon_{t+d-k_{3}} \epsilon_{t+d-k_{4}}\right)\right\} \\
& \{
\end{aligned}
$$

where the sums over $k_{u}, u=1, \ldots, 4$ are from 1 to $t-1$ (if $u=1$ or 2 ) or $t+d-1$ (if $u=3$ or 4 ). In the sequel, the sums that don't contain any term (because of the 
constraints imposed to the summation indices) are set equal to zero. As we did for the proof of $\mathbf{C}_{1}, \mathrm{~A} .14$ ) can be written

$$
\begin{gathered}
\sum_{k} \psi_{t i k} \psi_{t j k} \psi_{t+d, i, k+d} \psi_{t+d, j, k+d} E\left(\epsilon_{t-k}^{4}\right)+\sigma^{4}\left\{\sum_{\substack{k_{1} k_{3} \\
k_{1} \neq k_{3}-d}} \sum_{t i k_{1}} \psi_{t j k_{1}} \psi_{t+d, i, k_{3}} \psi_{t+d, j, k_{3}}\right. \\
\left.+\sum_{\substack{k_{1} \\
k_{1} \neq k_{2}}} \psi_{t i k_{1}} \psi_{t j k_{2}} \psi_{t+d, i, k_{1}+d} \psi_{t+d, j, k_{2}+d}+\sum_{\substack{k_{1} k_{2} \\
k_{1} \neq k_{2}}} \sum_{t i k_{1}} \psi_{t j k_{2}} \psi_{t+d, i, k_{2}+d} \psi_{t+d, j, k_{1}+d}\right\} \\
-\left\{\sum_{k_{1}} \sum_{k_{2}} \psi_{t i k_{1}} \psi_{t j k_{2}} E\left(\epsilon_{t-k_{1}} \epsilon_{t-k_{2}}\right)\right\}\left\{\sum_{k_{3}} \sum_{k_{4}} \psi_{t+d, i, k_{3}} \psi_{t+d, j, k_{4}} E\left(\epsilon_{t+d-k_{3}} \epsilon_{t+d-k_{4}}\right)\right\} .
\end{gathered}
$$

By the completion of the sums,

$$
\begin{aligned}
& \kappa \sum_{k} \psi_{t i k} \psi_{t j k} \psi_{t+d, i, k+d} \psi_{t+d, j, k+d} \\
& +\sigma^{4}\left[\left\{\sum_{k} \psi_{t i k} \psi_{t+d, i, k+d}\right\}\left\{\sum_{k} \psi_{t j k} \psi_{t+d, j, k+d}\right\}\right. \\
& \left.+\left\{\sum_{k} \psi_{t i k} \psi_{t+d, j, k+d}\right\}\left\{\sum_{k} \psi_{t j k} \psi_{t+d, i, k+d}\right\}\right] .
\end{aligned}
$$

By $\mathbf{A}_{5}-\mathbf{A}_{6}$, we have

$$
\frac{1}{n^{2}} \sum_{d=1}^{n-1} \sum_{t=1}^{n-d}(\mathrm{~A} .15)=O\left(\frac{1}{n}\right) .
$$

The checking of the conditions of Lemma A.1 is finished. Consequently,

$$
\frac{1}{n} \sum_{t=1}^{n}\left(\left.\left.\frac{\partial e_{t}(\theta)}{\partial \theta_{i}}\right|_{\theta=\theta^{0}} \frac{\partial e_{t}(\theta)}{\partial \theta_{j}}\right|_{\theta=\theta^{0}}-E_{\theta^{0}}\left(\frac{\partial e_{t}(\theta)}{\partial \theta_{i}} \frac{\partial e_{t}(\theta)}{\partial \theta_{j}}\right)\right) \rightarrow 0 \text { a.s.. }
$$

This implies that a.s.

$$
\begin{aligned}
\lim _{n \rightarrow \infty} \frac{1}{2 n} \sum_{t=1}^{n} E_{\theta^{0}}\left(\frac{\partial^{2} \alpha_{t}(\theta)}{\partial \theta_{i} \partial \theta_{j}} / F_{t-1}\right) & =\left.\left.\lim _{n \rightarrow \infty} \frac{1}{n} \sum_{t=1}^{n} \frac{\partial e_{t}(\theta)}{\partial \theta_{i}}\right|_{\theta=\theta^{0}} \frac{\partial e_{t}(\theta)}{\partial \theta_{j}}\right|_{\theta=\theta^{0}} \\
& =\lim _{n \rightarrow \infty} \frac{1}{n} \sum_{t=1}^{n} E_{\theta^{0}}\left(\frac{\partial e_{t}(\theta)}{\partial \theta_{i}} \frac{\partial e_{t}(\theta)}{\partial \theta_{j}}\right)=V_{i j}
\end{aligned}
$$

as defined in $\mathbf{A}_{4}$ as an element of a strictly definite positive matrix of constants, so $\mathrm{C}_{3}$ is satisfied.

Proof of $\mathbf{C}_{4}$. By the mean value theorem, we have

$$
\left.\sum_{t=1}^{n} \frac{\partial^{2} \alpha_{t}(\theta)}{\partial \theta_{i} \partial \theta_{j}}\right|_{\theta=\theta^{*}}-\left.\sum_{t=1}^{n} \frac{\partial^{2} \alpha_{t}(\theta)}{\partial \theta_{i} \partial \theta_{j}}\right|_{\theta=\theta^{0}}=\left.\sum_{l=1}^{r}\left(\theta_{l}^{*}-\theta_{l}^{0}\right) \sum_{t=1}^{n} \frac{\partial^{3} \alpha_{t}(\theta)}{\partial \theta_{i} \partial \theta_{j} \partial \theta_{l}}\right|_{\theta=\theta^{* *}},
$$

for $i, j=1, \ldots, m$, where $\theta^{* *}$ is a point on the line joining $\theta^{*}$ and $\theta^{0}$, with $\left\|\theta^{*}-\theta^{0}\right\|<\Delta$. 
Hence, we have an upper bound for the left-hand side of A.4

$$
\begin{gathered}
\lim _{n \rightarrow \infty} \sup _{\Delta \downarrow 0}(n \Delta)^{-1}\left\|\theta^{*}-\theta^{0}\right\|\left|\sum_{t=1}^{n} \frac{\partial^{3} \alpha_{t}(\theta)}{\partial \theta_{i} \partial \theta_{j} \partial \theta_{l}}\right|_{\theta=\theta^{* *}} \mid \\
\leq \lim _{n \rightarrow \infty} \sup _{\Delta \downarrow 0} \frac{1}{n}\left|\sum_{t=1}^{n} \frac{\partial^{3} \alpha_{t}(\theta)}{\partial \theta_{i} \partial \theta_{j} \partial \theta_{l}}\right|_{\theta=\theta^{* *}} \mid
\end{gathered}
$$

by continuity when $\left\|\theta^{*}-\theta^{0}\right\| \rightarrow 0$, and, to check A.4 , we need to prove that

$$
\lim _{n \rightarrow \infty} \frac{1}{n}\left|\sum_{t=1}^{n} \frac{\partial^{3} \alpha_{t}(\theta)}{\partial \theta_{i} \partial \theta_{j} \partial \theta_{l}}\right|_{\theta=\theta^{0}} \mid<\infty \quad \text { for } \quad i, j, l=1, \ldots, m .
$$

Given (3.19-3.21), the derivative of A.7) with respect to $\theta_{l}$, the expression involved in A.17) involves terms like $\left(\partial^{2} e_{t}(\theta) / \partial \theta_{i} \partial \theta_{j}\right)\left(\partial e_{t}(\theta) / \partial \theta_{l}\right)$ and $e_{t}(\theta)\left(\partial^{3} e_{t}(\theta) / \partial \theta_{i} \partial \theta_{j} \partial \theta_{l}\right)$. For $i, j, l=1, \ldots, m$, let us define the following two sequences of random variables:

$$
\begin{aligned}
Y_{t}^{i j l} & =\left.\left.\frac{\partial^{2} e_{t}(\theta)}{\partial \theta_{i} \partial \theta_{j}}\right|_{\theta=\theta^{0}} \frac{\partial e_{t}(\theta)}{\partial \theta_{l}}\right|_{\theta=\theta^{0}}-E_{\theta^{0}}\left[\frac{\partial^{2} e_{t}(\theta)}{\partial \theta_{i} \partial \theta_{j}} \frac{\partial e_{t}(\theta)}{\partial \theta_{l}}\right], \\
W_{t, 3}^{i j l} & =\left.e_{t} \frac{\partial^{3} e_{t}(\theta)}{\partial \theta_{i} \partial \theta_{j} \partial \theta_{l}}\right|_{\theta=\theta^{0}}-E_{\theta^{0}}\left[e_{t}(\theta) \frac{\partial^{3} e_{t}(\theta)}{\partial \theta_{i} \partial \theta_{j} \partial \theta_{l}}\right] .
\end{aligned}
$$

The arguments are more involved. Let us treat the case of $Y_{t}^{i j l}$ because the other one is simpler. We need the definition of $L_{2}$-mixingale sequences and a strong law of large numbers for them, the strong law of large numbers for a mixingale sequence (Hall \& Heyde, 1980, p. 41, Theorem 2.21).

Definition A.1. Let $\left\{w_{t}, t \geq 1\right\}$ be square-integrable random variables on a probability space $(\Omega, F, P)$ and $\left\{F_{t},-\infty<t<\infty\right\}$ be an increasing sequence of $\sigma$-fields of $F$. Then $\left\{w_{t}, F_{t}\right\}$ is a $L_{2}$-mixingale sequence if for sequences of non-negative constants $\psi_{\nu}$ and $c_{t}$ where $\psi_{\nu} \rightarrow 0$ as $\nu \rightarrow \infty$, we have (a) $E\left\{E\left(w_{t} \mid F_{t-\nu}\right)^{2}\right\}^{1 / 2} \leq \psi_{\nu} c_{t}$, and (b) $E\left\{\left(w_{t}-E\left(w_{t} \mid F_{t+\nu}\right)\right)^{2}\right\}^{1 / 2} \leq \psi_{\nu+1} c_{t}$.

Lemma A.2. If $\left\{w_{t}, F_{t}\right\}$ is a $L_{2}$-mixingale sequence, and if $n^{-2} \sum_{t=1}^{n} c_{t}^{2}<\infty$, as $n \rightarrow \infty$, and $\psi_{\nu}=O\left(\nu^{-1 / 2}(\log \nu)^{-2}\right)$ as $\nu \rightarrow \infty$, then $n^{-1} \sum_{t=1}^{n} w_{t} \stackrel{\text { a.s. }}{\rightarrow} 0$ as $n \rightarrow \infty$.

Let us show that $\left\{Y_{t}^{i j l}, F_{t}\right\}$ is a $L_{2}$-mixingale sequence, hence satisfies the two conditions of Definition A.1. To prove (a), an upper bound of $E\left\{E\left(Y_{t}^{i j l} / F_{t-\nu}\right)^{2}\right\}^{1 / 2}$ given in Lemma A.2 is obtained by first using an expression for $E\left\{E\left(Y_{t}^{i j l} / F_{t-\nu}\right)^{2}\right\}$. We replace $\left(\partial e_{t}(\theta) / \partial \theta_{l}\right)$ and $\left(\partial^{2} e_{t}(\theta) / \partial \theta_{i} \partial \theta_{j}\right)$ using, respectively, (3.19) and (3.20). Hence

$$
\begin{aligned}
Y_{t}^{i j l} & =\left.\frac{\partial e_{t}(\theta)}{\partial \theta_{l}} \frac{\partial^{2} e_{t}(\theta)}{\partial \theta_{i} \partial \theta_{j}}\right|_{\theta=\theta^{0}}-E_{\theta^{0}}\left(\frac{\partial e_{t}(\theta)}{\partial \theta_{l}} \frac{\partial^{2} e_{t}(\theta)}{\partial \theta_{i} \partial \theta_{j}}\right) \\
& =\sum_{k_{1}=1}^{t-1} \sum_{k_{2}=1}^{t-1} \psi_{t l k_{1}} \psi_{t i j k_{2}}\left\{\epsilon_{t-k_{1}} \epsilon_{t-k_{2}}-E\left(\epsilon_{t-k_{1}} \epsilon_{t-k_{2}}\right)\right\} .
\end{aligned}
$$


and

$$
E_{\theta^{0}}\left(Y_{t}^{i j l} / F_{t-\nu}\right)=\sum_{k_{1}=\nu+1}^{t-1} \sum_{k_{2}=\nu+1}^{t-1} \psi_{t l k_{1}} \psi_{t i j k_{2}}\left\{\epsilon_{t-k_{1}} \epsilon_{t-k_{2}}-E\left(\epsilon_{t-k_{1}} \epsilon_{t-k_{2}}\right)\right\},
$$

and, using the $L_{2}$-norm $\|\cdot\|_{2}$

$$
\begin{aligned}
& \left\|E_{\theta^{0}}\left(Y_{t}^{i j l} / F_{t-\nu}\right)\right\|_{2}^{2}=E\left\{E_{\theta^{0}}\left(Y_{t}^{i j l} / F_{t-\nu}\right)^{2}\right\} \\
= & \sum_{k_{1}=\nu+1}^{t-1} \sum_{k_{2}=\nu+1}^{t-1} \sum_{k_{3}=\nu+1}^{t-1} \sum_{k_{4}=\nu+1}^{t-1} \psi_{t l k_{1}} \psi_{t i j k_{2}} \psi_{t l k_{3}} \psi_{t i j k_{4}} \\
& E\left[\left\{\epsilon_{t-k_{1}} \epsilon_{t-k_{2}}-E\left(\epsilon_{t-k_{1}} \epsilon_{t-k_{2}}\right)\right\}\left\{\epsilon_{t-k_{3}} \epsilon_{t-k_{4}}-E\left(\epsilon_{t-k_{3}} \epsilon_{t-k_{4}}\right)\right\}\right],
\end{aligned}
$$

so that we have a quadruple sum, more or less as in (A.13), except that the lower summation index is $\nu+1$ instead of 1 . The treatment is similar:

$$
\begin{aligned}
\left\|E_{\theta^{0}}\left(Y_{t}^{i j l} / F_{t-\nu}\right)\right\|_{2}^{2} & =\sum_{\substack{k=\nu+1 \\
t-1}}^{t} \psi_{t l k}^{2} \psi_{t i j k}^{2} \operatorname{var}\left(\epsilon_{t-k}^{2}\right) \\
& +\sum_{\substack{k_{1} \\
k_{1} \neq k_{2}}} \sum_{t l k_{1}}^{2} \psi_{t i j k_{2}}^{2} E\left(\epsilon_{t-k_{1}}^{2}\right) E\left(\epsilon_{t-k_{2}}^{2}\right) \\
& +\sum_{\substack{k_{1} \\
k_{1} \neq k_{2}}} \sum_{k_{2}} \psi_{t l k_{1}} \psi_{t i j k_{2}} \psi_{t l k_{2}} \psi_{t i j k_{1}} E\left(\epsilon_{t-k_{1}}^{2}\right) E\left(\epsilon_{t-k_{2}}^{2}\right),
\end{aligned}
$$

which is bounded by a constant times $\Phi^{\nu}$ using $\mathbf{A}_{2}$. The second condition, concerning $\left\|Y_{t}^{i j l}-E_{\theta^{0}}\left(Y_{t}^{i j l} / F_{t+\nu}\right)\right\|_{2}$, is trivially true. Consequently $\left(Y_{t}^{i j l}, F_{t}\right)$ is an $L_{2}$-mixingale sequence, with the sequence $\Psi_{\nu}=\Phi^{\nu} \rightarrow 0$, exponentially as $\nu \rightarrow \infty$, since $0 \leq \Phi<1$, and the sequence of constants $c_{t}$ doesn't vary with $t$. Applying Lemma A.2 implies $n^{-1} \sum_{t=1}^{n} Y_{t}^{i j l} \rightarrow 0$ almost surely.

We can proceed still more simply for $W_{t, 3}^{i j l}$ defined in A.18 because the first factor $e_{t}(\theta)$ of the first term does not require the replacement by a sum and the other one is based on the development in (3.21).

Consequently, $\mathbf{C}_{4}$ is checked and we can conclude that the first four assumptions of Theorem A.1 are satisfied. Consequently, there exists an estimator $\hat{\theta}_{n}$ such that $\hat{\theta}_{n} \rightarrow \theta^{0}$ almost surely.

Proof of $\mathbf{C}_{5}$. Let us determine the explicit form of A.5 for $i, j=1, \ldots, m$. From A.6, it is equal to

$$
\frac{4}{n} \sum_{t=1}^{n}\left[\left.\left.\frac{\partial e_{t}(\theta)}{\partial \theta_{i}}\right|_{\theta=\theta^{0}} \frac{\partial e_{t}(\beta)}{\partial \theta_{j}}\right|_{\theta=\theta^{0}}-E_{\theta^{0}}\left(\frac{\partial e_{t}(\theta)}{\partial \theta_{i}} \frac{\partial e_{t}(\theta)}{\partial \theta_{j}}\right)\right] .
$$

While checking $H_{1.3}$, we have shown that (A.16), so $(\mathrm{A} .19)$ tends to zero.

As a conclusion, the asymptotic convergence of the estimator $\hat{\theta}_{n}$ toward the normal 
distribution is ensured and the proof of Theorem A.2 is achieved.

\section{A.4. Putting a tdARMA model in $t d V A R(1)$ form}

This section explains the changes that need to be made to the main paper Sections 3.1 and 3.2. Since we consider a scalar model, we have $r=1$ and most vectors and matrices in Section 3.1, such as $X_{t}(\theta), E_{t}(\theta), A_{t}(\theta), \tilde{B}_{t}(\theta), C, \tilde{A}_{t}(\theta), B_{t}(\theta), \tilde{C}$, $U_{1}$ and $U_{p+1}$ of the main paper, are ordinary vectors and matrices, not block vectors, or block matrices. More precisely, we have

$$
\begin{aligned}
& X_{t}(\theta)=\left(x_{t}, x_{t-1}, \ldots, x_{t-p+1}, e_{t}(\theta), e_{t-1}(\theta), \ldots, e_{t-q+1}(\theta)\right)^{T}, \\
& E_{t}(\theta)=\left(e_{t}(\theta), 0, \ldots, 0, e_{t}(\theta), 0, \ldots, 0\right)^{T} \text {, } \\
& A_{t}(\theta)=\left(\begin{array}{ccccc}
A_{1, t}(\theta) & A_{2, t}(\theta) & \ldots & A_{p-1, t}(\theta) & A_{p, t}(\theta) \\
1 & 0 & \ldots & 0 & 0 \\
0 & 1 & \ldots & 0 & 0 \\
\vdots & \vdots & \ddots & \vdots & \vdots \\
0 & 0 & \ldots & 1 & 0
\end{array}\right), \\
& \tilde{B}_{t}(\theta)=\left(\begin{array}{ccccc}
B_{1, t}(\theta) & B_{2, t}(\theta) & \ldots & B_{q-1, t}(\theta) & B_{q, t}(\theta) \\
0 & 0 & \ldots & 0 & 0 \\
0 & 0 & \ldots & 0 & 0 \\
\vdots & \vdots & \ddots & \vdots & \vdots \\
0 & 0 & \ldots & 0 & 0
\end{array}\right) \text {, } \\
& C=\left(\begin{array}{ccccc}
0 & 0 & \ldots & 0 & 0 \\
1 & 0 & \ldots & 0 & 0 \\
0 & 1 & \ldots & 0 & 0 \\
\vdots & \vdots & \ddots & \vdots & \vdots \\
0 & 0 & \ldots & 1 & 0
\end{array}\right) \\
& \tilde{A}_{t}(\theta)=\left(\begin{array}{ccccc}
-A_{1, t}(\theta) & -A_{2, t}(\theta) & \cdots- & A_{p-1, t}(\theta) & -A_{p, t}(\theta) \\
0 & 0 & \cdots & 0 & 0 \\
0 & 0 & \cdots & 0 & 0 \\
\vdots & \vdots & \ddots & \vdots & \vdots \\
0 & 0 & \cdots & 0 & 0
\end{array}\right) \text {, } \\
& B_{t}(\theta)=\left(\begin{array}{ccccc}
-B_{1, t}(\theta) & -B_{2, t}(\theta) & \ldots & -B_{q-1, t}(\theta) & -B_{q, t}(\theta) \\
1 & 0 & \ldots & & 0 \\
0 & 1 & \ldots & 0 & 0 \\
\vdots & \vdots & \ddots & \vdots & \vdots \\
0 & 0 & \ldots & 1 & 0
\end{array}\right) \text {, }
\end{aligned}
$$




$$
\begin{aligned}
& \tilde{C}=\left(\begin{array}{ccccc}
0 & 0 & \ldots & 0 & 0 \\
1 & 0 & \ldots & 0 & 0 \\
0 & 1 & \ldots & 0 & 0 \\
\vdots & \vdots & \ddots & \vdots & \vdots \\
0 & 0 & \ldots & 1 & 0
\end{array}\right) \\
& U_{p+1}=\left(\begin{array}{lllllll}
0 & \ldots & 0 & 1 & 0 & \ldots & 0
\end{array}\right)^{T},(\text { with } 1 \text { in position }(p+1)) \text {, } \\
& U_{1}=\left(\begin{array}{llll}
1 & 0 & \ldots & 0
\end{array}\right)^{T} \text {. }
\end{aligned}
$$

Otherwise, the relations at the end of Section 3.1 are identical, and all of Sections $3.2,3.4$, and 4.3 are unchanged. So we have the same expressions for the $\phi(\theta)$, $J \phi(\theta), \Psi_{t k}, \psi_{t k}, \Psi_{t i k}, \psi_{t i k}$, and so on.

\section{A.5. Verification of the assumptions for ARMA models}

Since we use the theory for a $\operatorname{tdVAR}(1)$ model, there are not many simplifications in this part.

The proofs of Lemma's 4.1, 4.3, and 4.4 cannot be simplified much because we still need to use Frobenius norms, except if $p+q=1$. As was mentioned in the text, however, the condition for identifiability is simpler and Lemmas 4.5 and 4.6 are not necessary: it is enough to suppose that the scalar autoregressive and moving average polynomials do not have a common root. Provided the causality and invertibility conditions are fulfilled, this is a necessary and sufficient condition for identifiability, and not merely a sufficient condition.

\section{Additional references}

Basawa, I. V. and Prakasa Rao, B. L. S. (1980). Statistical Inference for Stochastic Processes, Academic Press, London New York.

Hall, P. and Heyde, C. C. (1980). Martingale Limit Theory and its Application, Academic Press, New York.

Stout, W. F. (1974). Almost Sure Convergence, Academic Press, New York. 